\title{
CLUSTER CATEGORIES COMING FROM CYCLIC POSETS
}

\author{
KIYOSHI IGUSA AND GORDANA TODOROV
}

\begin{abstract}
Cyclic poset are generalizations of cyclically ordered sets. In this paper we show that any cyclic poset gives rise to a Frobenius category over any discrete valuation ring $R$. The continuous cluster categories of [13] are examples of this construction. If we twist the construction using an admissible automorphism of the cyclic poset, we generate other examples such as the $m$-cluster category of type $A_{\infty}(m \geq 3)$.
\end{abstract}

\section{Contents}

Introduction 1

1. General theory 3

1.1. Cyclic posets 3

1.2. Linearization of cyclic posets

1.3. Frobenius category 8

1.4. Twisted version 8

2. Cluster categories 10

2.1. Krull-Schmidt theorem 11

2.2. Frobenius cyclic posets 12

2.3. Continuous cluster categories 14

2.4. Discrete cluster category of type $A$

2.5. $m$-cluster category of type $A_{\infty} \quad$ 20]

References 28

\section{INTRODUCTION}

In this paper we combine two concepts which have appeared in recent liturature: $\mathbb{N}$ categories and matrix factorization categories in order to construct various triangulated categories including cluster categories of type $A$, continuous cluster categories and $m$-cluster categories of type $A_{\infty}$ for $m \geq 3$. Matrix factorization was introduced by Eisenbud [8] and developed by Buchweitz in an unpublished paper [4] (see also [5]), Orlov [19], 20] and many others [18, [7. $\mathbb{N}$-categories are a version of the $\mathbb{Z}$-categories considerer by Drinfeld [6] who, in turn, attributes it to Besser [2] and Grayson [9]. We take the multiplicative version which we call a $t^{\mathbb{N}}$-category, very similar to a construction which occurs in van Roosmalen [21]. We call the basic underlying structure a "cyclic poset."

Given any set $X$, we define a cyclic partial ordering of $X$ to be an equivalence class of posets $\tilde{X}$ together with a free $\mathbb{Z}$ action and a bijection $\tilde{X} / \mathbb{Z} \cong X$ with the additional

2010 Mathematics Subject Classification. 18E30:16G20.

The first author is supported by NSA Grant \#H98230-13-1-0247.

The second author is supported by NSF Grant \#DMS-1103813. 
property of being recurrent, i.e. that any $x, y \in X$ have liftings $\tilde{x}, \tilde{y} \in \tilde{X}$ so that $\tilde{x}<\tilde{y}$ (Definition 1.1.1). We observe that cyclic posets are special cases of $\mathbb{N}$-categories (Definition 1.1.15, taken essentially from [6], [21]). But we only consider these special cases.

Given a discrete valuation ring $R$, we define a $t^{\mathbb{N}}$-category $\mathcal{P}$ over $R$ to be a small $R$ category with two properties (Definition 1.2.1). The first is that $\mathcal{P}(x, y) \cong R$ for any two elements of the set of objects $X$ of $\mathcal{P}$. The second is that $\mathcal{P}(x, y)$ has a generator $f_{x y}$ with the property that, for any three $x, y, z \in X$,

$$
f_{y z} \circ f_{x y}=t^{n} f_{x z}
$$

where $n=c(x y z)$ is a nonnegative integer. We call $f_{x y}$ the "basic morphism" from $x$ to $y$. When $R=\mathbb{k}[[t]]$ is the power series ring in one variable over a field $\mathbb{k}$, the category $\mathcal{P}(X)$ can also be interpretted as the completed linearization of $X$ over $\mathbb{k}: \mathcal{P}(X) \cong \widehat{\mathbb{k} X}$ (Proposition 1.2.7).

The function $c: X^{3} \rightarrow \mathbb{N}$ is necessarily a reduced (2)-cocycle where "reduced" means $c(x x y)=0=c(x y y)$ for any $x, y \in X$ and the cocycle condition, equivalent to associativity of composition in $\mathcal{P}$, is

$$
c(x y z)-c(w y z)+c(w x z)-c(w x y)=0
$$

for any $w, x, y, z \in X$. Conversely, any reduced cocycle $c: X^{3} \rightarrow \mathbb{N}$ on any set $X$ defines a $t^{\mathbb{N}}$-category denoted $\mathcal{P}(X, c)$ or simply $\mathcal{P}(X)$. The structure of a (recurrent) cyclic partial ordering on any set $X$ is also determined by a uniquely determined reduced cocycle $c$ : $X^{3} \rightarrow \mathbb{N}$. Therefore, we can formally define a cyclic poset to be a pair $(X, c)$ where $c$ is a reduced cocycle on $X$.

Suppose $\phi$ is an admissible automorphisms $(X, c)$ (e.g., $\phi=i d_{X}$ : see Subsection 1.4). Then $\phi$ extends to an $R$-linear automorphism of $\mathcal{P}(X)$ and there is a natural transformation $\eta_{V}: V \rightarrow \phi(V)$ given on each component of $V$ by the basic morphism $x \rightarrow \phi(x)$. Let $\mathcal{F}_{\phi}(X)$ denote the category of all pairs $(V, d)$ where $V$ is an object of $\mathcal{P}(X)$ and $d$ is an endomorphism of $V$ satisfying the following two properties.

(1) $d^{2}=t$ is multiplication by $t$.

(2) $d$ factors through $\eta_{V}: V \rightarrow \phi(V)$.

If $\phi$ is admissible, then we show that $\mathcal{F}_{\phi}(X)$ is a Frobenius category. Let $\mathcal{C}_{\phi}(X)$ be the stable category of $\mathcal{F}_{\phi}(X)$. Then $\mathcal{C}_{\phi}(X)$ is a triangulated category.

The category $\mathcal{F}_{\phi}(X)$ is a matrix factorization category, not by definition but by our theorem which says that any object $(V, d)$ in $\mathcal{F}_{\phi}(X)$ decomposes in $\mathcal{P}(X)$ as a direct sum of two projective objects $V=V_{0} \oplus V_{1}$ so that $d: V \rightarrow V$ has the form

$$
d=\left[\begin{array}{cc}
0 & \beta \\
\alpha & 0
\end{array}\right]: \quad V_{0} \stackrel{\beta}{\longleftarrow} V_{1}
$$

In other words, $\alpha: V_{0} \rightarrow V_{1}, \beta: V_{1} \rightarrow V_{0}$ are morphisms so that $\alpha \beta=t$ and $\beta \alpha=t$. In the analogous case when $V_{0}, V_{1}$ are replaced by free modules over a commutative ring $R$, such morphisms are given by square matrices $A, B$ so that $A B=B A=t I_{n}$, i.e., $(A, B)$ is a matrix factorization of $t$ in the traditional sense. In a matrix factorization category, one usually assumes that objects are $\mathbb{Z} / 2$-graded. For us, the grading is undefined and there is no difference between even and odd morphisms.

In the remainder of the paper we explore examples and special cases of Frobenius categories of the form $\mathcal{F}_{\phi}(X)$ and their stable categories $\mathcal{C}_{\phi}(X)$. There include: 
(1) Continuous Frobenius categories over $R$

(2) Cluster categories of type $A_{n}$ over the field $\mathbb{k}=R /(t)$ where $1 \leq n \leq \infty$

(3) $m$-cluster categories of type $A_{\infty}$ over $\mathbb{k}$ for $m \geq 3$

This paper was partially written while the second author was at the Mathematical Sciences Research Institute (MSRI) in Berkeley.

\section{General theory}

1.1. Cyclic posets. We will carefully go over the "covering poset" description of a cyclic poset structure on a set $X$ and show that this cyclic poset structure is equivalent to choosing a reduced cocycle $c: X^{3} \rightarrow \mathbb{N}$. First, we recall that a poset is a set $X$ with a transitive, reflexive relation $\leq$. Two elements of $X$ are equivalent and we write $x \approx y$ if $x \leq y$ and $y \leq x$. We write $x<y$ if $x \leq y$ and $x \not z y$. A morphism of posets $f: X \rightarrow Y$ is a mapping which preserves the relation: $x \leq y \Rightarrow f(x) \leq f(y)$.

\subsubsection{Covering posets.}

Definition 1.1.1. A covering poset of set $X$ is a poset $\tilde{X}$ together with a surjective mapping $\pi: \tilde{X} \rightarrow X$ satisfying the following.

(1) The inverse image $\pi^{-1}(x) \subseteq \tilde{X}$ of any $x \in X$ is isomorphic as a poset to the set of integers with the usual ordering.

(2) There exists an automorphism $\sigma$ of the poset $\tilde{X}$ so that, for any $\tilde{x} \in \tilde{X}, \sigma \tilde{x}$ is the smallest element of $\pi^{-1}(\pi(\tilde{x}))$ which is greater than $\tilde{x}$.

(3) For every pair $\tilde{x}, \tilde{y} \in \tilde{X}$ there exists an integer $m \geq 0$ so that $\tilde{x} \leq \sigma^{m} \tilde{y} \leq \sigma^{2 m} \tilde{x}$.

An equivalence of covering posets $f: \tilde{X} \rightarrow \tilde{X}^{\prime}$ is a $\sigma$-equivariant poset isomorphism over $X$, i.e., $\pi^{\prime} f=\pi$ and $\sigma f=f \sigma$.

Remark 1.1.2. In the above definition, the automorphism $\sigma$ of the covering poset $\tilde{X}$ is uniquely determined.

Example 1.1.3. The simplest example of this is a set with $n$ elements $Z_{n}=\{1,2, \cdots, n\}$. One covering poset of $Z_{n}$ is given by the set of integers $\tilde{Z}_{n}=\mathbb{Z}$ with the usual ordering and the automorphism $\sigma x=x+n: \tilde{X}: \quad \cdots<1<2<\cdots<n<\sigma 1<\sigma 2<\cdots$.

1.1.2. Cocycles. We will show that covering posets over $X$ are classified by reduced cocycles $c: X^{3} \rightarrow \mathbb{N}$ as defined below.

Definition 1.1.4. A cocycle on $X$ is a function $c: X^{3} \rightarrow \mathbb{N}$, written $c(x y z)$, so that

$$
\delta c(w x y z):=c(x y z)-c(w y z)+c(w x z)-c(w x y)=0
$$

for all $w, x, y, z \in X$. We say that $c$ is reduced if $c(x x y)=c(x y y)=0$ for all $x, y \in X$.

Example 1.1.5. Central group extensions $\mathbb{Z} \rightarrow E \rightarrow G$ are classified by elements of $H^{2}(G ; \mathbb{Z})$ and represented by factor sets $f: G^{2} \rightarrow \mathbb{Z}$ which may be taken to be reduced in the sense that $f(g, h)=0$ if $g=1$ or $h=1$. If this reduced factor set happens to have only nonnegative values, it gives a reduced cocycle $c$ by the formula $c(x y z)=f\left(x^{-1} y, y^{-1} z\right)$. Every such cocycle gives a distinct cyclic poset structure on the underlying set of $G$.

Example 1.1.6. A cyclic ordering on a set $X$ is the same as a reduced cocycle on $X$ with values 0,1 where $c(x y z)=0$ if and only if either $x, y, z \in X$ are in cyclic order with $x \not z z$ or $x \approx y \approx z$. 
Definition 1.1.7. Given a covering poset $\pi: \tilde{X} \rightarrow X$ and a section $\lambda: X \rightarrow \tilde{X}$, we define the corresponding distance function $b_{\lambda}: X^{2} \rightarrow \mathbb{Z}$ by letting $b_{\lambda}(x y)=m$ be the smallest integer so that $\lambda(x) \leq \sigma^{m} \lambda(y)$.

We note that $b_{\lambda}$ is reduced in the sense that $b_{\lambda}(x x)=0$ for all $x \in X$.

Lemma 1.1.8. Let $\pi: \tilde{X} \rightarrow X$ be a covering poset and $\lambda$ a section of $\pi$. Then

$$
c(x y z):=\delta b_{\lambda}(x y z)=b_{\lambda}(y z)-b_{\lambda}(x z)+b_{\lambda}(y z)
$$

is a reduced cocycle $c: X^{3} \rightarrow \mathbb{N}$ and $c$ is independent of the choice of sections $\lambda: X \rightarrow \tilde{X}$.

Proof. Since $\delta^{2}=0$, it follows that $c$ is a cocycle. Also, $c$ is easily seen to be reduced. To prove uniqueness, suppose that $\lambda^{\prime}$ is another section of $\pi$. Then $\lambda^{\prime}(x)=\sigma^{a(x)} \lambda(x)$ for some function $a: X \rightarrow \mathbb{Z}$. Then the two distance function are related by $b_{\lambda^{\prime}}(x y)=$ $b_{\lambda}(x y)-a(y)+a(x)$ or $b_{\lambda^{\prime}}=b_{\lambda}-\delta a$. So, $\delta b_{\lambda^{\prime}}=\delta b_{\lambda}-\delta^{2} a=\delta b_{\lambda}$ as claimed.

Remark 1.1.9. Since $c$ is uniquely determined, we refer to it as the structure cocycle of the covering poset $\pi: \tilde{X} \rightarrow X$

We say that $x, y \in X$ are equivalent and we write $x \approx y$ if $c(x y x)=c(y x y)=0$. It is easy to see that this is an equivalence relation and that $x \approx y$ if and only if $\tilde{x} \approx \tilde{y}$ for some liftings $\tilde{x}, \tilde{y}$ of $x, y$ to $\tilde{X}$.

Lemma 1.1.10. Given any reduced cocycle $c: X^{3} \rightarrow \mathbb{N}$ on any set $X$, there exists a covering poset $\tilde{X} \rightarrow X$ whose structure cocycle is c. Furthermore, $\tilde{X}$ is unique up to poset isomorphism over $X$.

Proof. Choose a base point $x_{0} \in X$. Then we can express $c$ as $c=\delta b$ where $b: X^{2} \rightarrow \mathbb{Z}$ is given by $b(x y)=c\left(x_{0} x y\right)$. A covering poset $\tilde{X}$ can now be given as follows. As a set, let $\tilde{X}=X \times \mathbb{Z}$ with $\sigma$ action given by $\sigma(x, i)=(x, i+1)$. Then the partial ordering of $\tilde{X}$ is given by: $(x, j) \leq(y, k)$ if $k \geq j+b(x y)$. In other words, $b(x y)$ is the distance function for the lifting $\tilde{x}=(x, 0)$. Therefore the structure cocycle of $\tilde{X}$ is $\delta b=c$.

If $\tilde{X}^{\prime} \rightarrow X$ is another covering poset with structure cocycle $c$ then a $\sigma$-equivariant poset isomorphism $f: X \times \mathbb{Z} \rightarrow \tilde{X}^{\prime}$ is given as follows. Choose a fixed lifting $\tilde{x}_{0} \in \tilde{X}^{\prime}$. Then for each $x \in X$ let $\tilde{x} \in \tilde{X}^{\prime}$ be the unique lifting of $x$ so that $\tilde{x}_{0} \leq \tilde{x}$ but $\sigma \tilde{x}_{0} \not \leq \tilde{x}$. Then, for any $x, y \in X$, the smallest integer $j$ so that $\tilde{x} \leq \sigma^{j} \tilde{y}$ is $j=c\left(x_{0} x y\right)=b(x y)$. Therefore, the covering posets $\tilde{X}^{\prime}$ and $X \times \mathbb{Z}$ have the same distance function which implies that they are isomorphic with isomorphism $f: X \times \mathbb{Z} \rightarrow \tilde{X}^{\prime}$ given by $f(x, j)=\sigma^{j} \tilde{x}$.

This proves the following theorem.

Theorem 1.1.11. For any set $X$, there is a 1-1 correspondence between equivalence classes of covering posets over $X$ and reduced cocycles $c: X^{3} \rightarrow \mathbb{N}$.

Intuitively, a cyclic poset structure on a set $X$ is an equivalence class of covering posets $\tilde{X} \rightarrow X$. However, because of the above theorem, we use the following simpler definition.

Definition 1.1.12. A cyclic poset is defined to be a pair $(X, c)$ where $X$ is a set and $c: X^{3} \rightarrow \mathbb{N}$ is a reduced cocycle on $X$.

Example 1.1.13. The product of cyclic posets $X_{1} \times X_{2}$ has reduced cocycle $c(x y z):=$ $c_{1}\left(x_{1} y_{1} z_{1}\right)+c_{2}\left(x_{2} y_{2} z_{2}\right)$ where $c_{1}, c_{2}$ are the cocycles of $X_{1}, X_{2}$ and $x=\left(x_{1}, x_{2}\right)$, etc. 
Example 1.1.14. Suppose that $X$ is a cyclic poset with covering poset $\tilde{X}$ and $P$ is another poset. Then another cyclic poset $X * P$ with underlying set $X \times P$ can be constructed as follows. The covering poset $\widetilde{X * P}$ of $X * P$ is the Cartesian product $\tilde{X} \times P$ with lexicographic order. So, $(\tilde{x}, p) \leq(\tilde{y}, q)$ if either $\tilde{x}<\tilde{y}$ or $\tilde{x} \approx \tilde{y}$ and $p \leq q$. The $\sigma$ action is given only on the first coordinate: $\sigma(\tilde{x}, p)=(\sigma \tilde{x}, p)$. An important example is $P=\mathbb{Z}$. If $X$ is cyclically ordered, then so is $X * \mathbb{Z}$.

1.1.3. $\mathbb{N}$-categories. Following, van Roosmalen 1011.6077, p.10 and Drinfeld 0304064, p.5, (who refers to Besser and Greyson), we note that a cyclic poset structure on a set $X$ is equivalent to a special case of an $\mathbb{N}$-category structure on (the object set) $X$.

Definition 1.1.15. An $\mathbb{N}$-category is a category $\mathcal{X}$ with the property that the additive monoid $\mathbb{N}$ acts freely on every Hom set

$$
\mathbb{N} \times \mathcal{X}(x, y) \rightarrow \mathcal{X}(x, y)
$$

so that composition satisfies:

$$
n f \circ m g=(n+m) f g: x \rightarrow z
$$

(Acting freely means Hom sets are disjoint unions of copies of $\mathbb{N}: \mathcal{X}(x, y)=\coprod \mathbb{N} f_{i}$.)

Proposition 1.1.16. A cyclic poset structure on a set $X$ is the same as an $\mathbb{N}$ category $\mathcal{X}$ with object set $X$ so that every Hom set $\mathcal{X}(x, y)$ is freely generated by one morphism $f_{x y}$.

Note that, given three objects, $x, y, z \in X$, we have

$$
f_{y z} f_{x y}=n f_{x z}
$$

for some $n \in \mathbb{N}$.

1.2. Linearization of cyclic posets. From now on we assume that $R$ is a discrete valuation ring with a fixed uniformizer $t$. We will construct the linearization of any cyclic poset $X=(X, c)$ over $R$.

\subsection{1. $t^{\mathbb{N}}$-categories.}

Definition 1.2.1. A $t^{\mathbb{N}}$-category over $R$ is defined to be a small category $\mathcal{P}$ with the following two properties:

(1) $\mathcal{P}(x, y)$ is a free $R$-module with one generator $f_{x y}$ for all $x, y$ in the object set $X$ of $\mathcal{P}$. Thus $\mathcal{P}(x, y)=\left\{r f_{x y} \mid r \in R\right\}$.

(2) For any objects $x, y, z \in X$, there is a nonnegative integer $n$ so that for all $r, s \in R$,

$$
r f_{y z} \circ s f_{x y}=r s t^{n} f_{x z} .
$$

Note that this is an $R$-category: Hom sets are $R$-modules and composition is $R$-bilinear. Note that the integer $n$ in the above equation is uniquely determined since $t^{n}=t^{m}$ in $R$ if and only if $n=m$. So, $n$ is a well defined element of $\mathbb{N}$ which depends only on $x, y, z$.

Lemma 1.2.2. Given a $t^{\mathbb{N}}$-category $\mathcal{P}$ over $R$, there is a unique reduced cocycle $c: X^{3} \rightarrow \mathbb{N}$ on its set of objects $X$ so that $f_{y z} f_{x y}=t^{c(x y z)} f_{x z}$ for all $x, y, z \in X$.

Proof. We have already remarked that $n$ gives a well-defined function $X^{3} \rightarrow \mathbb{N}$ which we will denote by $c$. It remains to show that $c$ is a reduced cocycle. 
First note that, if $r f_{x x}$ is the identity map on $x \in X$ then, for any $y \in X$, we must have $f_{x y}=f_{x y}\left(r f_{x x}\right)=r t^{c(x x y)} f_{x y}$. Therefore, we must have $r=1$ and $c(x x y)=0$. Similarly, $c(x y y)=0$. So, $c$ is reduced. Associativity of composition gives

$$
\begin{aligned}
& \left(f_{y z} f_{x y}\right) f_{w x}=t^{c(x y z)} f_{x z} f_{w x}=t^{c(x y z)+c(w x z)} f_{w z} \\
= & f_{y z}\left(f_{x y} f_{w x}\right)=t^{c(w x y)} f_{y z} f_{w y}=t^{c(w x y)+c(w y z)} f_{w z} .
\end{aligned}
$$

Therefore, $\delta c=c(x y z)+c(w x z)-c(w x y)-c(w y z)=0$. Thus $c$ is a reduced cocycle.

Definition 1.2.3. We call $(X, c)$ the underlying cyclic poset of the $t^{\mathbb{N}}$-category $\mathcal{P}$.

Example 1.2.4. Suppose that $b: X^{2} \rightarrow \mathbb{N}$ is a distance function for $c: X^{3} \rightarrow \mathbb{N}$ in the sense that $c=\delta b$. Let $\mathcal{P}$ denote the $R$-category with object set $X$ and morphism sets $\mathcal{P}(x, y)=$ $\left(t^{b(x y)}\right)$, the ideal in $R$ generated by $t^{b(x y)}$, with composition given by multiplication. Then composition of any morphisms $x \rightarrow y \rightarrow z$ will be divisible by $t^{b(x y)+b(y z)}=t^{c(x y z)+b(x z)}$ and therefore will lie in the ideal $\left(t^{b(x z)}\right)$ as required. Then $\mathcal{P}$ is a $t^{\mathbb{N}}$-category with underlying cyclic poset $(X, c)$.

By an isomorphism of cyclic posets $(X, c) \cong\left(X^{\prime}, c^{\prime}\right)$ we mean a bijection $x \leftrightarrow x^{\prime}$ so that $c(x y z)=c^{\prime}\left(x^{\prime} y^{\prime} z^{\prime}\right)$ for all $x, y, z \in X$.

Proposition 1.2.5. (a) Given any cyclic poset $(X, c)$, there is a $t^{\mathbb{N}}$-category $R X$ with underlying cyclic poset $(X, c)$.

(b) Any $t^{\mathbb{N}}$-category $\mathcal{P}$ is isomorphic to $R X$ if and only if its underlying cyclic poset is isomorphic to $(X, c)$.

Proof. (a) The $t^{\mathbb{N}}$-category $R X$ is given as follows. We take $X$ to be the object set of $R X$ but we denote by $P_{x}$ the object in $R X$ corresponding to $x \in X$. For any $x, y \in X$ we take $R X\left(P_{x}, P_{y}\right) \cong R$ to be the free $R$ module generated by the single element $f_{x y}$. Composition of morphisms $P_{x} \rightarrow P_{y} \rightarrow P_{z}$ is given by $\left(r f_{y z}\right) \circ\left(s f_{x y}\right)=r s t^{c(x y z)} f_{x z}$. As in the proof of the lemma above, composition is associative since $c$ is a cocycle and $f_{x x}$ is the identity map on $x$ since $c$ is reduced.

(b) Suppose we have a $t^{\mathbb{N}}$-category $\mathcal{P}$ with underlying cyclic poset $\left(X^{\prime}, c^{\prime}\right)$ and an isomorphism $\Phi: R X \rightarrow \mathcal{P}$. Then, $\Phi$ gives a bijection between the object set $X$ of $R X$ and the object set $X^{\prime}$ of $\mathcal{P}$ which we denote $P_{x} \mapsto x^{\prime}$. The basic morphism $f_{x y}: P_{x} \rightarrow P_{y}$ maps to a generator $r_{x y} f_{x^{\prime} y^{\prime}} \in \mathcal{P}\left(x^{\prime}, y^{\prime}\right)$. So, $r_{x y}$ must be a unit in $R$. Since $\Phi$ sends the identity $f_{x x}$ on $P_{x}$ to the identity on $x^{\prime}$, we must have $r_{x x}=1$. Finally, the relation $f_{y z} f_{x y}=t^{c(x y z)} f_{x z}$ gives the relation

which implies that

$$
r_{y z} r_{x y} f_{y^{\prime} z^{\prime}} f_{x^{\prime} y^{\prime}}=r_{x z} t^{c(x y z)} f_{x^{\prime} z^{\prime}}
$$

$$
r_{y z} r_{x y} t^{c^{\prime}\left(x^{\prime} y^{\prime} z^{\prime}\right)}=r_{x z} t^{c(x y z)}
$$

Since the $r$ 's are units, we conclude that $c^{\prime}\left(x^{\prime} y^{\prime} z^{\prime}\right)=c(x y z)$ for all $x, y, z \in X$.

Conversely, if the underlying cyclic poset $\left(X^{\prime}, c^{\prime}\right)$ of $\mathcal{P}$ is isomorphic to $(X, c)$ then we get a bijection $x^{\prime} \leftrightarrow P_{x}$ on the set of objects and this extends to the morphism sets by mapping $f_{x^{\prime} y^{\prime}}$ to $f_{x y}$. The cyclic poset isomorphism implies that the composition laws correspond. Therefore, we have an isomorphism of categories $\mathcal{P} \cong R X$.

In the special case when $R=\mathbb{k}[[t]]$, the $t^{\mathbb{N}}$-category $R X$ has another description as the completed orbit category of $\mathbb{k}_{k} \tilde{X}$. This idea comes from [21]. First, we recall that, since $\tilde{X}$ is a poset, it has a linearization $\mathbb{k} \tilde{X}$ over $\mathbb{k}$ defined as follows. The objects of $\mathbb{k} \tilde{X}$ are the 
elements of $\tilde{X}$ and hom sets $\mathbb{k} \tilde{X}(x, y)$ are either $\mathbb{k}$ or 0 depending on whether $x \leq y$ or not. Composition of morphisms is given by multiplication by scalars. The $\sigma$-orbit category of $\mathbb{k} \tilde{X}$ is the category whose objects are the $\sigma$-orbits $\bigoplus \sigma^{n} x \in A d d \mathbb{k} \tilde{X}$ and whose morphisms are $\mathbb{k}$-linear morphisms $f: \bigoplus \sigma^{n} x \rightarrow \bigoplus \sigma^{m} y$ which are $\sigma$-equivariant in the sense that $f \circ \sigma=\sigma \circ f$. This is the same as saying that the $(m, n)$ component of the morphism $f$ is equal to the $(m+i, n+i)$ component of $f$ for all $i$. Note that the $(m, n)$ component of $f$ can only be nonzero if $\sigma^{m} y \geq \sigma^{n} x$.

Definition 1.2.6. We define the completed linearization $\widehat{\mathbb{k} X}$ of a cyclic poset $X$ with covering poset $\pi: \tilde{X} \rightarrow X$ to be the following category. The objects of $\widehat{\mathbb{k} X}$ are the elements of $X$ and a morphism $f: x \rightarrow y$ is defined to be an infinite matrix $f=\left(f_{b a}\right)$ with entries $f_{b a} \in \mathbb{k}$ and indexed over all $a \in \pi^{-1}(x), b \in \pi^{-1}(y)$ so that

(1) $f_{b a}=0$ if $b \nsupseteq a$.

(2) $f_{b a}=f_{\sigma(b) \sigma(a)}$ for all $a, b$.

Composition of morphisms: $f \circ g=h$ is given by

$$
h_{c a}=\sum_{b \in \pi^{-1}(y)} f_{c b} g_{b a}
$$

Proposition 1.2.7. When $R=\mathbb{k}[[t]]$, there is a natural isomorphism of categories $R X \cong$ $\widehat{\mathbb{k} X}$.

Proof. To construct the isomorphism, we need to choose a fixed section $\lambda: X \rightarrow \tilde{X}$ and let $b_{\lambda}: X^{2} \rightarrow \mathbb{Z}$ be the corresponding distance function. Then, $c=\delta b_{\lambda}$ and

$$
\sigma^{i} \lambda(x) \leq \sigma^{j} \lambda(y) \Longleftrightarrow j-i-b_{\lambda}(x, y) \geq 0 .
$$

An isomorphism $\Theta: R X \rightarrow \widehat{\mathbb{k} X}$ is given as follows. On objects, $\Theta$ is the identity map: $\Theta x=x$ for all $x \in X$. On each morphism $g=\sum g_{n} t^{n} f_{x y}: x \rightarrow y$ in $R X, g_{n} \in \mathbb{k}$, we let $\Theta g: x \rightarrow y$ be the morphism in $\widehat{\mathbb{k} X}$ given by the matrix whose $\left(\sigma^{j} \lambda(y), \sigma^{i} \lambda(x)\right)$-entry is $g_{n}$ where $n=j-i-b_{\lambda}(x, y)$. Since this describes all possible morphisms $x \rightarrow y$ in $\widehat{\mathbb{k} X}, \Theta$ gives a bijection on object sets and a linear isomorphism of Hom sets. It remains only to show that $\Theta$ is a functor.

To see this, take another morphism $h=\sum h_{m} t^{m} f_{y z}: y \rightarrow z$ in $R X$. Then the matrix of $\Theta h$ has $\left(\sigma^{k} \lambda(z), \sigma^{j} \lambda(y)\right)$-entry equal to $h_{m}$ where $m=k-j-b_{\lambda}(y, z)$. The product of these two matrices has $\left(\sigma^{k} \lambda(z), \sigma^{i} \lambda(x)\right)$-entry equal to $\sum h_{m} g_{n}$ where the sum is over all $n, m$ so that

$$
n+m=k-i-b_{\lambda}(x, y)-b_{\lambda}(y, z)
$$

The composition of the morphisms $g, h$ in $R X$ is equal to

$$
h \circ g=\sum h_{m} g_{n} t^{m+n} f_{y z} f_{x y}=\sum h_{m} g_{n} t^{m+n+c(x y z)} f_{x z}
$$

Then $\Theta(h g)$ has $\left(\sigma^{k} \lambda(z), \sigma^{i} \lambda(x)\right)$-entry equal to $\sum h_{m} g_{n}$ where the sum is over all $n, m$ so that

$$
n+m+c(x y z)=k-i-b_{\lambda}(x, z)
$$

However, this is equivalent to 1.2 since $c=\delta b_{\lambda}$. Thus $\Theta$ commutes with composition. It also takes $i d_{x}$ to $i d_{x}$. So, $\Theta$ is a functor and therefore an isomorphism of categories. 
1.3. Frobenius category. For any cyclic poset $X$, let $\mathcal{P}(X)=\operatorname{add} R X$ denote the additive $R$-category generated by $R X$.

Definition 1.3.1. Let $\mathcal{M F}(X)$ denote the category of all pairs $(P, d)$ where $P \in \mathcal{P}(X)$ and $d: P \rightarrow P$ so that $d^{2}=\cdot t$ (multiplication by $t$ ). Morphism $f:(P, d) \rightarrow(Q, d)$ are maps $f: P \rightarrow Q$ so that $d f=f d$.

The key property is the adjunction lemma:

Lemma 1.3.2. The functor $G: \mathcal{P}(X) \rightarrow \mathcal{M F}(X)$ given by

$$
G P:=\left(P \oplus P,\left[\begin{array}{ll}
0 & t \\
1 & 0
\end{array}\right]\right): \quad P \overbrace{i d}^{\cdot t} P
$$

is both left and right adjoint to the forgetful functor $F: \mathcal{M F}(X) \rightarrow \mathcal{P}(X)$.

For the proof, see the twisted version below.

Theorem 1.3.3. For any cyclic poset $X, \mathcal{M F}(X)$ is a Frobenius category where a sequence

$$
(A, d) \longmapsto(B, d) \rightarrow(C, d)
$$

is defined to be exact in $\mathcal{M F}(X)$ if $A \longmapsto B \rightarrow C$ is (split) exact in $\mathcal{P}(X)$. Objects isomorphic to $G P$ for some $P$ in $\mathcal{P}$ are the projective injective objects of $\mathcal{M F}(X)$.

The twisted version of this theorem is proved in the next section. The fact that GP is projective and injective follows from the adjunction lemma.

Example 1.3.4. The main example of this construction is the continuous Frobenius category $\mathcal{M F}\left(S^{1}\right)$ which comes from the cyclically ordered set $S^{1}$. The stable category is the continuous cluster category $\underline{\mathcal{M F}}\left(S^{1}\right)=\mathcal{C}_{\pi}$. These are topological categories. The topology is used to define the cluster structure on $\mathcal{C}_{\pi}$, namely, two objects $X, Y$ are compatible if the ordered pair $(X, Y)$ lies in the closure of the set of all $\left(X^{\prime}, Y^{\prime}\right)$ satisfying $\operatorname{Ext}^{1}\left(X^{\prime}, Y^{\prime}\right)=0=\operatorname{Ext}^{1}\left(Y^{\prime}, X^{\prime}\right)$ and a cluster is defined to be a maximal set of pairwise compatible indecomposable objects which is also a discrete set (every element lies in an open set containing no other objects of the cluster). See [13], [14] for details.

To obtain other kinds of examples, we need to take a twisted version $\mathcal{M F}_{\phi}(X)$ of the Frobenius category.

1.4. Twisted version. We need to modify the above construction using an admissible automorphism of a cyclic poset.

An automorphism $\phi$ of $(X, c)$ will be called admissible if it is covered by a $\sigma$-equivariant order preserving bijection $\tilde{\phi}$ of the covering poset $\tilde{X}$ to itself satisfying the property:

$$
x \leq \tilde{\phi} x \leq \tilde{\phi}^{2} x<\sigma x
$$

for all $x \in \tilde{X}$. There is a corresponding additive $R$-linear automorphism of $\mathcal{P}(X)$, which we also call $\phi$, defined on indecomposable objects by $\phi P_{x}=P_{\phi x}$ and on basic morphisms by $\phi f_{x y}=f_{\phi x, \phi y}$. In $\mathcal{P}(X)$ the condition above gives morphisms

$$
P_{x} \stackrel{\eta_{x}}{\longrightarrow} \phi P_{x}=P_{\phi x} \stackrel{\xi_{x}}{\longrightarrow} P_{x}
$$

giving natural transformations

$$
P \stackrel{\eta_{P}}{\longrightarrow} \phi P \stackrel{\xi_{P}}{\longrightarrow} P
$$


whose composition $\xi_{P} \circ \eta_{P}: P \rightarrow P$ is multiplication by $t$. Since $t$ is not a zero divisor, neither are $\xi_{P}$ nor $\eta_{P}$. Therefore, the reverse composition $\eta_{P} \circ \xi_{P}: \phi P \rightarrow \phi P$ is also multiplication by $t$ since $\xi \eta \xi=t \xi=\xi t$ making $\xi(\eta \xi-t)=0$.

Definition 1.4.1. Let $\mathcal{M F}_{\phi}(X)$ be the full subcategory of $\mathcal{M F}(X)$ of all $(P, d)$ where $d: P \rightarrow P$ factors through $\eta_{P}: P \rightarrow \phi P$.

An example of an object of $\mathcal{M F}_{\phi}(X)$ is given by

$$
G_{\phi} P:=\left(P \oplus \phi P,\left[\begin{array}{cc}
0 & \xi_{P} \\
\eta_{P} & 0
\end{array}\right]\right):
$$

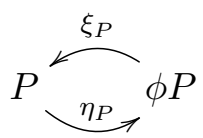

We will show that $\mathcal{M F}_{\phi}(X)$ is a Frobenius category with projective-injective objects given by $G_{\phi} P$. We use the key observation that $\eta_{P}, \xi_{P}$ are not zero divisors. This implies that, for any object $(P, d)$ in $\mathcal{M F}_{\phi}(X)$, the factorization

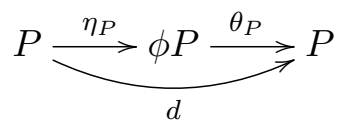

is unique. Similarly, $d \theta_{P}=\xi_{P}: \phi P \rightarrow P$ since $d \theta_{P} \eta_{P}=d^{2}=\cdot t=\xi_{P} \eta_{P}$.

Furthermore, for all morphisms $f:(V, d) \rightarrow(W, d)$, the following commutes.

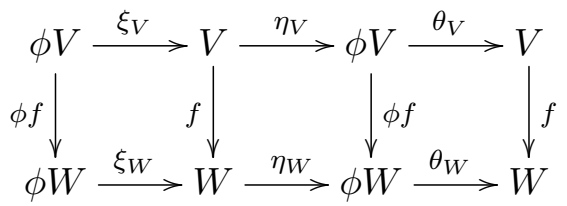

This is because $\eta_{V}$ is not a zero divisor: $\left(f \theta_{V}-\theta_{W} \phi f\right) \eta_{V}=f d-\theta_{W} \eta_{W} f=f d-d f=0$ implies $f \theta_{V}=\theta_{W} \phi f$. Note that the left two squares in the diagram commute for any morphism $f: V \rightarrow W$ in $\mathcal{P}(X)=a d d R X$ since $\xi, \eta$ are natural transformations.

Lemma 1.4.2. $\mathcal{M F}_{\phi}(X)$ is an exact category where a sequence

$$
(A, d) \longmapsto(B, d) \rightarrow(C, d)
$$

in $\mathcal{M F}_{\phi}(X)$ is defined to be exact if the underlying sequence $A \rightarrow B \rightarrow C$ is split exact in $\mathcal{P}(X)$.

The proof is straighrforward and identical to the case of $X=S^{1}$ detailed in [13.

Lemma 1.4.3. The functor $G_{\phi}: \mathcal{P}(X) \rightarrow \mathcal{M F}_{\phi}(X)$ is left adjoint to the forgetful functor $\mathcal{M F}_{\phi}(X) \rightarrow \mathcal{P}(X)$ and the functor $G_{\phi} \circ \phi^{-1}$ is right adjoint to the forgetful functor. In other words, we have natural isomorphisms:

$$
\mathcal{M F}_{\phi}\left(G_{\phi} V,(W, d)\right) \cong \mathcal{P}(V, W) \cong \mathcal{M F}_{\phi}\left((V, d), G_{\phi} \phi^{-1} W\right)
$$

Proof. A morphism $f: V \rightarrow W$ corresponds to the morphisms $\left(f, \theta_{W} \phi f\right): G_{\phi} V \rightarrow(W, d)$ and $\left(\phi^{-1}\left(f \theta_{V}\right), f\right):(V, d) \rightarrow G_{\phi} \phi^{-1} W$. This is verified by a calculation in which we should remember that $\theta_{W} \phi f \neq f \theta_{V}$. However, since $d$ commutes with itself, we have $\theta_{V} \phi d=d \theta_{V}$.

For example, to prove right adjunction, we need to verify that $\phi^{-1}\left(f \theta_{V}\right) d=\left(\xi_{\phi^{-1} W}\right) f$ and $\eta_{\phi^{-1} W} \phi^{-1}\left(f \theta_{V}\right)=f d$. This follows from:

$$
f \theta_{V} \phi d=f d \theta_{V}=f \xi_{V}=\xi_{W} \phi f
$$


Applying $\phi^{-1}$ to both sides gives $\phi^{-1}\left(f \theta_{V}\right) d=\phi^{-1}\left(\xi_{W}\right) f=\xi_{\phi^{-1} W} f$ since $\xi$ is natural. Also

$$
\left(\eta_{W} f \theta_{V}\right) \eta_{V}=\eta_{W} f d=\phi f \eta_{V} d=(\phi f \phi d) \eta_{V}
$$

Cancel $\eta_{V}$ from both sides and apply $\phi^{-1}$ to get $\eta_{\phi^{-1} W} \phi^{-1}\left(f \theta_{V}\right)=f d$.

Verification of left adjunction is similar but easier.

Proposition 1.4.4. If $P=P_{x}$ is indecomposable in $\mathcal{P}(X)$ then the endomorphism ring of $G_{\phi} P_{x}$ is isomorphic to the local ring $R[u]$ where $u^{2}=t$.

Proof. Morphisms $G_{\phi} V \rightarrow(W, d)$ are given by $\left(f, \theta_{W} \phi f\right)$ where $f: V \rightarrow W$ is any morphism in $\mathcal{P}(X)$. If $V=P_{x}$ and $(W, d)=G_{\phi} P_{x}$ then $W=P_{x} \oplus \phi P_{x}$ and $f: P_{x} \rightarrow P_{x} \oplus \phi P_{x}$ is a sum of two morphisms $f_{0}=r_{0} f_{x x}: P_{x} \rightarrow P_{x}$ and $f_{1}=r_{1} f_{x \phi x}: P_{x} \rightarrow \phi P_{x}$ where $r_{0}, r_{1} \in R$. Since $\theta_{W}=i d_{x} \oplus \xi_{x}^{\prime}$ we get the following formula for a general endomorphism of $G_{\phi} P_{x}$ :

$$
\left(f, \theta_{W} \phi f\right)=\left[\begin{array}{cc}
f_{0} & \xi_{x}^{\prime} \phi f_{1} \\
f_{1} & \phi f_{0}
\end{array}\right]=\left[\begin{array}{cc}
r_{0} f_{x x} & t r_{1} f_{\phi x x} \\
r_{1} f_{x \phi x} & r_{0} f_{\phi x \phi x}
\end{array}\right]
$$

Since the second column is determined by the first, we can write this as $\left(r_{0}, r_{1}\right)$. Then composition is given by $\left(r_{0}, r_{1}\right)\left(s_{0}, s_{1}\right)=\left(r_{0} s_{0}+t r_{1} s_{1}, r_{1} s_{0}+r_{0} s_{1}\right)$ which is exactly the multiplication rule for $\left(r_{0}, r_{1}\right)=r_{0}+r_{1} u$ in $R[u]$ where $u^{2}=t$.

Corollary 1.4.5. Every object $G_{\phi} V$ can be expressed uniquely up to isomorphism as a direct sum of indecomposable objects $G_{\phi} P_{x_{i}}$ where $P_{x_{i}}$ are the components of $V$.

Proof. If $V=\bigoplus P_{x_{i}}$ then $G_{\phi} V=\bigoplus G_{\phi} P_{x_{i}}$ where each $G_{\phi} P_{x_{i}}$ is indecomposable.

Theorem 1.4.6. $\mathcal{M F}_{\phi}(X)$ is a Frobenius category with projective-injective objects given by $G_{\phi} V$ for all $V$ in $\mathcal{P}(X)$.

Proof. By the lemma above, $G_{\phi} V$ is projective for all $V$ and $G_{\phi} \phi^{-1} W$ is injective for all $W$. To see this note that $\mathcal{M F}_{\phi}\left(G_{\phi} V,-\right)=\mathcal{P}(V, F(-))$ and $\mathcal{M F}_{\phi}\left(-, G_{\phi} \phi^{-1} W\right)=\mathcal{P}(F(-), W)$ are exact since the forgetful functor $F: \mathcal{M F}_{\phi}(X) \rightarrow \mathcal{P}(X)$ takes exact sequences to split exact sequences. Letting $W=\phi V$ we see that $G_{\phi} V$ is projective and injective for all $V$.

For every $(V, d)$ there is a quotient map $G_{\phi} V \rightarrow(V, d)$ adjoint to the identity map on $V$. Similarly there is a cofibration $(V, d) \rightarrow G_{\phi} \phi^{-1} V$. Thus, there are enough projectiveinjective objects. If $(V, d)$ is projective or injective these morphisms must split, making $(V, d)$ a summand of either $G_{\phi} V$ or $G_{\phi} \phi^{-1} V$. By the corollary, this implies $(V, d)$ is a direct sum of $G_{\phi} P_{x_{i}}$. Since $\mathcal{M F}_{\phi}(X)$ is an exact category with enough projectives all of which are also injective, it is a Frobenius category by definition.

\section{Cluster categories}

Recall that the stable category $\underline{\mathcal{F}}$ of a Frobenius category $\mathcal{F}$ has the same set of objects as $\mathcal{F}$ with morphism sets:

$$
\underline{\mathcal{F}}(A, B)=\frac{\mathcal{F}(A, B)}{A \rightarrow P \rightarrow B, P \text { proj-inj }}
$$

Theorem 2.0.7 (Happel [10]). The stable category of a Frobenius category is triangulated.

We denote the stable categories of $\mathcal{M F}(X)$ and $\mathcal{M F}_{\phi}(X)$ by $\mathcal{C}(X)=\underline{\mathcal{M F}}(X)$ and $\mathcal{C}_{\phi}(X)=\underline{\mathcal{M F}}_{\phi}(X)$. These triangulated categories will have a cluster structure if $X$ and $\phi$ are carefully chosen. Cluster categories were first constructed by Buan-Marsh-ReinekeReiten-Todorov (0402054) as orbit categories. This construction is an alternate construction in type $A$. 
2.1. Krull-Schmidt theorem. If $X$ is cyclically ordered we will show that $\mathcal{M F}(X)$ is Krull-Schmidt $R$-category with indecomposable objects $E(x, y)$ defined below with $x, y \in$ $X$. This will imply that $\mathcal{C}(X)$ is a Krull-Schmidt $\mathbb{k}$-category with indecomposable objects $E(x, y)$ where $y \not z x$. The following easy proposition implies that $\mathcal{M F}_{\phi}(X)$ and thus $\mathcal{C}_{\phi}(X)$ will also be Krull-Schmidt categories.

Proposition 2.1.1. The subcategory $\mathcal{M F}_{\phi}(X)$ is closed under direct summands in $\mathcal{M F}(X)$.

Proof. Suppose that $\left(P_{1}, d_{1}\right) \oplus\left(P_{2}, d_{2}\right)$ lies in $\mathcal{M F}_{\phi}(X)$. Then $d_{1} \oplus d_{2}: P_{1} \oplus P_{2} \rightarrow P_{1} \oplus P_{2}$ factors through $\eta_{1} \oplus \eta_{2}: P_{1} \oplus P_{2} \rightarrow \phi P_{1} \oplus \phi P_{2}$. Then $d_{1}$ factors through $\eta_{1}$ and $d_{2}$ factors through $\eta_{2}$ making $\left(P_{1}, d_{1}\right)$ and $\left(P_{2}, d_{2}\right)$ objects of $\mathcal{M F}_{\phi}(X)$.

To construct the objects $E(x, y)$, we first recall that $c(x y x)=c(y x y)=0$ if and only if $x \approx y$ if and only if $P_{x} \cong P_{y}$.

Definition 2.1.2. We define a sequence of three not necessarilty distinct elements $(x, y, z)$ in $X$ to be in cyclic order if there exist liftings $\tilde{x}, \tilde{y}, \tilde{z} \in \tilde{X}$ (possibly $\tilde{x} \neq \tilde{z}$ when $x=z$ ) so that $\tilde{x} \leq \tilde{y} \leq \tilde{z} \leq \sigma \tilde{x}$. For example, $\left(x, \phi x, \phi^{2} x\right)$ is in cyclic order for any admissible automorphism $\phi$ of $X$.

It is easy to see that, if $(x y z),(z w x)$ are in cyclic order and $x \not \approx z$ then $(y z w)$ is also in cyclic order since there is a lifting $\tilde{w} \in \tilde{X}$ of $w$ so that $\tilde{x}<\tilde{z} \leq \tilde{w} \leq \sigma^{n} \tilde{x}<\sigma \tilde{z}<\sigma^{2} \tilde{x}$ forcing $n=1$. We call this composition of cyclic order. When the entire set $X$ is cyclically ordered, $(x y z)$ is in cyclic order if and only if either $c(x y z)=0$ or $x \approx z$.

Definition 2.1.3. Let $(X, c)$ be a cyclic poset and let $x, y \in X$ so that $c(x y x)=c(y x y)=1$. Then we define $E(x, y)$ to be the object in $\mathcal{M F}(X)$ given by

$$
E(x, y):=\left(P_{x} \oplus P_{y},\left[\begin{array}{cc}
0 & f_{y x} \\
f_{x y} & 0
\end{array}\right]\right) .
$$

If $x \approx y$ then we define $E(x, y)$ and $E(x, y)^{\prime}$ by

$$
E(x, y):=\left(P_{x} \oplus P_{y},\left[\begin{array}{cc}
0 & t f_{y x} \\
f_{x y} & 0
\end{array}\right]\right), \quad E(x, y)^{\prime}:=\left(P_{x} \oplus P_{y},\left[\begin{array}{cc}
0 & f_{y x} \\
t f_{x y} & 0
\end{array}\right]\right)
$$

For example, $E(x, \phi x)=\left(P_{x} \oplus P_{\phi x},\left[\begin{array}{cc}0 & \xi_{x} \\ \eta_{x} & 0\end{array}\right]\right)=G_{\phi} P_{x}$ in $\mathcal{M F}_{\phi}(X)$ is the projectiveinjective object in $E(x, \phi x)$.

We observe that $E(x, y)^{\prime} \cong E(y, x)$ by the isomorphism which switches $P_{x}, P_{y}$. This second copy of the same object is convenient for notational symmetry and is used in the proof of Corollary 2.2.2 below. (In the notation of [13], [14], $E(x, x)^{\prime}=E(x, x+2 \pi)$.)

Lemma 2.1.4. $E(x, y)$ is isomorphic to $E(a, b)$ in $\mathcal{M F}(X)$ if and only if they are isomorphic in $\mathcal{P}(X)$. In particular, $E(x, y) \cong E(y, x)$.

Proof. We only need to prove sufficiency. Suppose that $P_{x} \oplus P_{y} \cong P_{a} \oplus P_{b}$. Then either $x \approx a$ and $y \approx b$ in $X$ or $x \approx b$ and $y \approx a$. In the first case, it is clear that $E(x, y) \cong E(a, b)$. In the second case we have $x \not \approx y$ (otherwise we are in Case 1 ). Then the transposition isomorphism $P_{x} \oplus P_{y} \cong P_{y} \oplus P_{x}$ commutes with the operator $d$ and therefore gives an isomorphism $E(x, y) \cong E(y, x)$. But $E(y, x) \cong E(a, b)$ as in Case 1. So, $E(x, y) \cong E(a, b)$ in both cases.

Lemma 2.1.5. The endomorphism ring of $E(x, y)$ is isomorphic to the local ring $R[\sqrt{t}]$. 
Proof. This is true for $y=\phi x$ by Proposition 1.4 .4 and the same proof works for any $x, y$. Alternatively, note that the condition $c(x y x) \leq 1$ implies that the cyclic subposet $\{x, y\}$ of $X$ is cyclically ordered and therefore can be embedded in the circle $S^{1}$. Then the endomorphism ring of $E(x, y)$ is isomorphic to the endomorphism ring of the corresponding object of $\mathcal{M F}\left(S^{1}\right)$ which was shown to have endomorphism ring $R[\sqrt{t}]$ in [13].

Lemma 2.1.6. The object $E(x, y)$ of $\mathcal{M F}(X)$ lies in $\mathcal{M F}_{\phi}(X)$ if and only if $\left(\phi x, y, \phi^{-1} x\right)$ is in cyclic order.

Proof. Suppose that $E(x, y)$ lies in $\mathcal{M F}_{\phi}(X)$. Then the condition that $f_{x y}: P_{x} \rightarrow P_{y}$ factors through $\eta: P_{x} \rightarrow P_{\phi x}$ is equivalent to the condition that $c(x, \phi x, y)=0$. The other condition, that $t^{n} f_{y x}: P_{y} \rightarrow P_{x}$ factors through $\eta: P_{y} \rightarrow P_{\phi y}$ is equivalent to either $x \approx y$ or $x \not \approx y$ and $c\left(y, \phi^{-1} x, x\right)=0$. In the second case we conclude that $\left(\phi x, y, \phi^{-1} x\right)$ is in cyclic order. In the first case we must have $x \approx \phi x \approx y$ which also implies that $\left(\phi x, y, \phi^{-1} x\right)$ is in cyclic order.

Conversely, suppose that $\left(\phi x, y, \phi^{-1} x\right)$ is in cyclic order. Then, either $\phi x \approx \phi^{-1} x$, which implies $x \approx \phi x$ making $c(x, \phi x, y)=0=c\left(y, \phi^{-1} x, x\right)$, so that $E(x, y)$ lies in $\mathcal{M F}_{\phi}(X)$ or $\phi x \not \phi^{-1} x$, which implies that $x \not \approx y$ and, since $\left(\phi^{-1} x, x, \phi x\right)$ is in cyclic order, it also implies that $(x, \phi x, y)$ and $\left(y, \phi^{-1} x, x\right)$ are in cyclic order by composition of cyclic order.

Theorem 2.1.7. Suppose that $(X, c)$ is cyclically order and $\phi$ is an admissible automorphism of $X$. Then every object of $\mathcal{M F}_{\phi}(X)$ is isomorphic to a finite direct sum of objects of the form $E(x, y)$ where $\left(\phi x, y, \phi^{-1} x\right)$ is in cyclic order. Furthermore, each $E(x, y)$ is indecomposable and the indecomposable direct summands of any object of $\mathcal{M F}_{\phi}(X)$ are uniquely determined up to isomorphism. Finally, $E(x, y)$ is projective injective in $\mathcal{M F}_{\phi}(X)$ if and only if either $y \approx \phi x$ or $x \approx \phi y$.

Proof. We use the fact that any finite cyclically order set $X_{0}$ has a cyclic order preserving embedding $X_{0} \hookrightarrow S^{1}$ and the fact that the theorem holds for $X=S^{1}$ and $\phi=i d_{S^{1}}$ by [13]. If $(P, d)$ is any object of $\mathcal{M F}_{\phi}(X)$ then $P=\bigoplus P_{x_{i}}$. Let $X_{0}=\left\{x_{i}\right\}$. Since this is a finite cyclically ordered set, we conclude that $(P, d)$, as an object of $\mathcal{M F}(X)$, decomposes into objects $E(x, y)$ with $x, y \in X_{0}$. By Proposition 2.1.1, the components of $(P, d)$ also lie in $\mathcal{M F}_{\phi}(X)$. So, $\left(\phi x, y \phi^{-1} x\right)$ is in cyclic order by Lemma 2.1.6 above.

The uniqueness of decomposition follows from Lemma 2.1.5.

By Theorem 1.4.6, the indecomposable projective-injective objects of $\mathcal{M F}_{\phi}(X)$ are $G_{\phi} P_{z}=E(z, \phi z)$. By Lemma 2.1.4 the object $E(x, y)$ is isomorphic to such an object if and only if either $y \approx \phi x$ or $x \approx \phi y$.

2.2. Frobenius cyclic posets. We need the following corollaries and definitions as the starting points of our next two papers $([15],[16])$.

Definition 2.2.1. Suppose that $Z$ is a cyclically ordered set with totally ordered covering poset $\tilde{Z}$ and $\phi$ is an admissible automorphism of $Z$ with corresponding covering automorphism $\tilde{\phi}: \tilde{Z} \rightarrow \tilde{Z}$ so that $a \leq \tilde{\phi} a<\sigma \tilde{\phi}^{-1} a \leq \sigma a$ for all $a \in \tilde{Z}$. Then we define $\mathcal{X}(Z, \phi)$ to be the cyclic poset given as follows. The covering poset of $\mathcal{X}(Z, \phi)$ is the set

$$
\tilde{\mathcal{X}}=\left\{(a, b) \in \tilde{Z} \times \tilde{Z} \mid \tilde{\phi} a \leq b \leq \sigma \tilde{\phi}^{-1} a\right\}
$$

with partial ordering $(a, b) \leq\left(a^{\prime}, b^{\prime}\right)$ if and only if $a \leq a^{\prime}$ and $b \leq b^{\prime}$ and with automorphism $\sigma$ defined by $\sigma(a, b)=(b, \sigma a)$. Let $\mathcal{X}_{0}(Z, \phi)$ be the cyclic subposet of $\mathcal{X}(Z, \phi)$ given by the covering subposet

$$
\tilde{\mathcal{X}}_{0}=\left\{(a, b) \mid b \approx \phi a \text { or } b \approx \phi^{-1} a\right\} .
$$


Corollary 2.2.2. Let $R=\mathbb{k}[[t]]$. Then the additive $R$-category $\mathcal{P}(\mathcal{X}(Z, \phi))=$ add $R \mathcal{X}$ has the structure of a Frobenius category with $\mathcal{P}\left(\mathcal{X}_{0}(Z, \phi)\right)$ being the full subcategory of projective-injective objects.

In other words, the pair $\left(\mathcal{X}(Z, \phi), \mathcal{X}_{0}(Z, \phi)\right)$ satisfies the following definition.

Definition 2.2.3. A Frobenius cyclic poset is defined to be a pair $\left(X, X_{0}\right)$ where $X$ is a cyclic poset and $X_{0}$ is a subset of $X$ with the induced cyclic poset structure such that, for $R=\mathbb{k}[[t]]$ with $\mathbb{k}$ any field, $\mathcal{P}(X)=\operatorname{add} R X$ has the structure of a Frobenius category with projective injective objects forming the full subcategory $\mathcal{P}\left(X_{0}\right)$.

We will see in [15] that the exact structure of such a Frobenius category is uniquely determined. So the words "has the structure of a Frobenius category" in the above definition can be replaced with "has a uniquely determined structure of a Frobenius category".

In order to prove the corollary it will be useful to "double" the cyclic poset $\mathcal{X}$ since there are two objects in the Frobenius category $\mathcal{M F}_{\phi}\left(Z, R_{0}\right)$ (where $R_{0}=\mathbb{k}\left[\left[t^{2}\right]\right]$ ) for every object in $R \mathcal{X}$.

Definition 2.2.4. For $Z, \tilde{Z}, \phi, \tilde{\phi}$ as in Definition 2.2.1, let $\mathcal{X}^{(2)}=\mathcal{X}^{(2)}(Z)$ denote the cyclic poset given by the covering poset $\tilde{\mathcal{X}}^{(2)}$ which consists of two copies of $\tilde{\mathcal{X}}$ :

$$
\tilde{\mathcal{X}}^{(2)}=\tilde{\mathcal{X}} \times\{+,-\}=\left\{(a, b)_{\epsilon} \mid a, b \in \tilde{Z}, \epsilon= \pm, \tilde{\phi} a \leq b \leq \sigma \tilde{\phi}^{-1} a\right\}
$$

with partial ordering disregarding the sign: $(a, b)_{\epsilon} \leq\left(a^{\prime}, b^{\prime}\right)_{\epsilon^{\prime}}$ if $a \leq a^{\prime}$ and $b \leq b^{\prime}$. In particular, $(a, b)_{+} \approx(a, b)_{-}$. The automorphism $\sigma$ is given by $\sigma(a, b)_{\epsilon}=(b, \sigma a)_{-\epsilon}$. Let $\mathcal{X}^{(2)}$ be the set of $\sigma$-orbits in $\tilde{\mathcal{X}}^{(2)}$ and let $\mathcal{X}_{0}^{(2)}$ be the subset of $\sigma$-orbits of $\tilde{\mathcal{X}}_{0}^{(2)}=\tilde{\mathcal{X}}_{0} \times\{ \pm\}$.

Clearly, $R \mathcal{X}^{(2)}$ is equivalent to $R \mathcal{X}$ and this equivalence sends $R \mathcal{X}_{0}^{(2)}$ to $R \mathcal{X}_{0}$.

Proof of Corollary 2.2.2. Let $R_{0}=\mathbb{k}\left[\left[t^{2}\right]\right]$. This is a discrete valuation ring with uniformizer $v=t^{2}$ and $R=R_{0}[\sqrt{v}]$. Let $\mathcal{M F}_{\phi}\left(Z, R_{0}\right)$ be the Frobenius category constructed from the cyclic poset $Z$ using $R_{0}, v$ instead of $R, t$ in Definitions 1.3.1, 1.4.1. Let $\mathcal{M}(Z)$ be the full subcategory of $\mathcal{M F}_{\phi}\left(Z, R_{0}\right)$ with objects $E(x, y)$ (where $\left(\phi x, y, \phi^{-1} x\right)$ are in cyclic order) and $E(x, y)^{\prime}$ (where $x \approx \phi x \approx y$ ) and let $\mathcal{M}_{0}(Z)$ be the full subcategory of $\mathcal{M}(Z)$ of the projective-injective objects $E(x, y)$ (where either $y \approx \phi x$ or $x \approx \phi y$ ) and $E(x, y)^{\prime}$ (where $x \approx \phi x \approx y)$.

We claim that $\mathcal{M}(Z)$ is isomorphic to $R \mathcal{X}^{(2)}$ as $R$-categories and that this isomorphism sends $\mathcal{M}_{0}(Z)$ to $R \mathcal{X}_{0}^{(2)}$. This will imply that the additive categories that they generate are also equivalent and this equivalence will give the structure of a Frobenius category to add $R \mathcal{X}^{(2)}$ with $a d d R \mathcal{X}_{0}^{(2)}$ being the full subcategory of projective-injective objects, thereby proving the corollary.

A bijection between the objects of $\mathcal{M}(Z)$ and the objects of $R \mathcal{X}^{(2)}$ which are the elements of $\mathcal{X}^{(2)}$ is given as follows. Let $\psi: \tilde{\mathcal{X}}^{(2)} \rightarrow \operatorname{Ob\mathcal {M}}(Z)$ be defined by

$$
\psi(x, y)_{+}= \begin{cases}E(\pi(x), \pi(y))^{\prime} & \text { if } y \approx \sigma \tilde{\phi} x \approx \sigma x \\ E(\pi(x), \pi(y)) & \text { otherwise }\end{cases}
$$

and $\psi(x, y)_{-}=\psi(y, \sigma x)_{+}$. Then we claim that $\psi$ induces a bijection $\Psi$ between the set of $\sigma$-orbits in $\tilde{\mathcal{X}}^{(2)}$ and the set of objects of $\mathcal{M}(Z)$. The inverse of $\Psi$ is given as follows. If $x \not \approx y$ then $\Psi^{-1} E(x, y)$ is the $\sigma$-orbit of $(\tilde{x}, \tilde{y})+$ where $\tilde{x}, \tilde{y} \in \tilde{Z}$ are any lifting of $x, y$ so that $\tilde{\phi} \tilde{x} \leq \tilde{y} \leq \tilde{\phi}^{-1} \tilde{x}$. If $x \approx y$ then this is not well defined since there are two such $\sigma$ orbits 
given by $(\tilde{x}, \tilde{y})_{+}$and $(\tilde{x}, \sigma \tilde{y})_{+}$where $\tilde{x} \approx \tilde{y}$. In this case we let $\Psi^{-1} E(x, y)=(\tilde{x}, \tilde{y})_{+}$and $\Psi^{-1} E(x, y)^{\prime}=(\tilde{x}, \sigma \tilde{y})_{+}$. It is straightforward to show that these are inverse maps.

We will use the completed linearization $\widehat{\mathbb{k}_{\boldsymbol{X}}(2)}$ instead of $R \mathcal{X}^{(2)}$ since they are isomorphic. (Definition 1.2.6 and Proposition 1.2.7) We also use the fact that $\widehat{\mathbb{k} Z} \cong R_{0} Z$. An isomorphism $\Psi: \widehat{\mathbb{k}_{k} \mathcal{X}^{(2)}} \rightarrow \mathcal{M}(Z)$ is given on object above and on morphisms below.

Since morphisms on both sides are given by infinite series of monomials, we will describe the isomorphism only on monomial morphisms. There are two kinds of monomial morphisms: even and odd. An even monomial morphism, call it $f$, from the $\sigma$ orbit of $(x, y)_{+}$ to that of $(a, b)_{+}$is given by a scalar $r$ times inequalities $\left(\sigma^{n} x, \sigma^{n} y\right)_{+} \leq\left(\sigma^{n+i} a, \sigma^{n+i} b\right)_{+}$ and $\left(\sigma^{n} y, \sigma^{n+1} x\right)_{-} \leq\left(\sigma^{n+i} b, \sigma^{n+i+1} a\right)_{-}$for all $n$ but fix $i$ and an odd morphism, call it $g$, between the same two $\sigma$-orbits is given by a scalar $s$ times the inequalities $\left(\sigma^{n} x, \sigma^{n} y\right)_{+} \leq$ $\left(\sigma^{n+j} b, \sigma^{n+j+1} a\right)_{-}$and $\left(\sigma^{n} y, \sigma^{n+1} x\right)_{-} \leq\left(\sigma^{n+j+1} a, \sigma^{n+j+1} b\right)_{+}$for all $n$ but fixed $j$.

The functor $\Psi$ takes the $\sigma$-orbit of $(x, y)_{+}$to $\Psi(x, y)_{+}=\left(P_{\pi(x)} \oplus P_{\pi(y)}, d\right)$ where $d$ is the counterdiagonal matrix whose entries are given, as morphisms in $\widehat{\mathbb{k} Z}$, by the inequalities $x \leq y$ and $y \leq \sigma x$. The functor $\Psi$ takes the even morphism $f$ to the diagonal morphism $\Psi(x, y)_{+} \rightarrow \Psi(a, b)_{+}$given by the $2 \times 2$ matrix whose off-diagonal entries are zero and whose diagonal entries are $r$ times the morphism $\pi(x) \rightarrow \pi(a)$ in $\widehat{\mathbb{k} Z}$ given by the inequalities $\sigma^{n} x \leq \sigma^{n+i} a$ for all $n$ and $r$ times the morphism $\pi(y) \rightarrow \pi(b)$ given by $\sigma^{n} y \leq \sigma^{n+i} b$ for all $n$. The functor $\Psi$ also takes the odd morphism $g$ to the matrix whose diagonal entries are zero and whose off-diagonal entries are $s$ times the inequalities $\sigma^{n} x \leq \sigma^{n+j} b$ and $\sigma^{n} y \leq \sigma^{n+j+1} a$. The functor $\Psi$ commutes with composition since composition is defined component-wise on both sourse and target. I.e., $\Psi$ lies over the isomorphism add $\widehat{\mathbb{k} Z} \rightarrow$ add $R_{0} Z$. Therefore, $\Psi$ gives an isomorphism of categories $\Psi: \widehat{\mathbb{k} \mathcal{X}^{(2)}} \cong \mathcal{M}(Z)$.

2.3. Continuous cluster categories. In this family of examples, $X$ is the cyclically ordered set $X=S^{1}=\mathbb{R} / 2 \pi \mathbb{Z}$ with covering poset $\tilde{X}=\mathbb{R}$ with $\sigma(x)=x+2 \pi$. For any fixed $0 \leq \theta<\pi$, let $\phi$ be the automorphism of $S^{1}$ with lifting $\tilde{\phi}(x)=x+\theta$. Then $\phi$ is admissible and the Frobenius category $\mathcal{M F}_{\phi}\left(S^{1}\right)$ is equal to the continuous Frobenius category $\mathcal{F}_{\pi-\theta}$ with stable category $\mathcal{C}_{\pi-\theta}$. (See [13, 14].) In subsequent papers [15, 16] we use the fact (Corollary 2.2.2 that, for $R=\mathbb{k}[[t]]$ and $R_{0}=\mathbb{k}\left[\left[t^{2}\right]\right]$, the Frobenius category $\mathcal{M F}_{\phi}\left(S^{1}, R_{0}\right)$ is isomorphic to the completed linearization of the Frobenius cyclic poset $\left(\mathcal{X}, \mathcal{X}_{0}\right)$ where

$$
\tilde{\mathcal{X}}=\left\{(x, y)_{\epsilon} \in \mathbb{R}^{2} \times\{ \pm\} \mid x+\theta \leq y \leq x+2 \pi-\theta\right\}
$$

partially ordered by $(x, y)_{\epsilon} \leq\left(x^{\prime}, y^{\prime}\right)_{\epsilon^{\prime}}$ if $x \leq x^{\prime}$ and $y \leq y^{\prime}$ and $\sigma(x, y)_{\epsilon}=(y, x+2 \pi)_{-\epsilon}$.

The indecomposable objects of $\mathcal{C}_{\pi-\theta}$ are $E(x, y)$ where $x, y$ are distinct points on the circle subtending an angle more than $\theta$. This triangulated category has a cluster structure if and only if $\theta=2 \pi /(n+3)$ for some positive integer $n$ (Cor. 5.4.4 in [14]).

2.4. Discrete cluster category of type $A$. The basic examples of discrete cluster categories of type $A$ are given by the cyclic posets $Z_{n}=\{1,2 \cdots, n\}$ where $n \geq 3$ with $\phi(i)=i+1$ modulo $m$ which give the cluster category of type $A_{n-3}$ and the cyclic poset $\mathbb{Z}$, with $\phi(i)=i+1$, which gives the $\infty$-gon of [11]. Both are examples of the following construction.

Theorem 2.4.1. Let $Z$ be a cyclically ordered set having at least four elements with totally ordered covering $\tilde{Z}$ and let $\phi$ be an admissible automorphism of $Z$ satisfying the following.

(1) $x<\tilde{\phi} x<\tilde{\phi}^{2} x<\sigma x$ for all $x \in \tilde{Z}$. 
(2) There are no elements $z \in \tilde{Z}$ so that $x<z<\tilde{\phi} x$ for any $x \in \tilde{Z}$.

Then the stable category of the Frobenius category $\mathcal{M F}_{\phi}(Z)$ is a 2-Calabi-Yau cluster category.

This is a mild generalization of the results of [17], [11. We refer to [11 for many of the proofs. We will give the definitions and statements so that the reader can make the comparison and we give one new example: when $Z=\mathbb{Z}^{2}$ with lexicographic order.

2.4.1. 2-Calabi-Yau property. For convenience of notation we assume that $Z$ is infinite and that no two distinct elements of $Z$ are equivalent. Then we can take $Z$ to be a totally ordered set and $\phi$ to be an automorphism of $Z$ so that $\phi x$ is the smallest element of $Z$ which is greater than $x$ for every $x \in Z$. We call $\phi x$ the successor of $x$ and denote it by $x^{+}$. Similarly, we call $\phi^{-1} x$ the predecessor of $x$ and we denote it by $x^{-}$.

The indecomposable objects of $\mathcal{M F}_{\phi}(Z)$ are $E(x, y) \cong E(y, x)$ where $x, y$ are distinct elements of $Z$. We denote the one with first coordinate small than the second by the set $X=\{x, y\}$. (Thus $X=E(x, y)$ if $x<y$.) Such a subset $X$ will denote a projective-injective object if and only if the larger element of $X$ is the successor of the smaller element.

Finally, we observe that the stable category of $\mathcal{M F}_{\phi}(Z)$ depends only on $\mathbb{k}=R / \mathfrak{m}$ and is independent of the choice of $R$ since multiplication by $t$ always factors through a projective-injective object. Therefore, we may assume that $R=\mathbb{k}[[t]]$ and $\mathcal{M F}_{\phi}(Z)=\mathcal{P}(\mathcal{X})$ and $\mathcal{C}_{\phi}(Z)=\mathcal{P}(\mathcal{X}) / \mathcal{P}\left(\mathcal{X}_{0}\right)$ with $\mathcal{X}=\mathcal{X}(Z), \mathcal{X}_{0}=\mathcal{X}_{0}(Z)$ given by Definition 2.2.1. Since $t=0$ in the stable category, $\mathcal{C}_{\phi}(X, Y)=\mathbb{k}$ or 0 for all indecomposable objects $X, Y$.

Lemma 2.4.2. If $X, Y$ are 2-element subsets of $Z$ which are not projective-injective then, in the stable category $\mathcal{C}_{\phi}(Z)$ of $\mathcal{M F}_{\phi}(Z)$, we have $\mathcal{C}_{\phi}(Z)(X, Y)=\mathbb{k}$ if

$$
x_{0} \leq y_{0}<x_{1}^{-}, \quad x_{1} \leq y_{1}<\sigma x_{0}^{-}
$$

for some liftings $x_{i}, y_{i} \in \tilde{Z}$ of the elements of $X, Y$ and $\mathcal{C}_{\phi}(Z)(X, Y)=0$ otherwise. Furthermore, any nonzero morphism $X \rightarrow Y$ factors through a 2-element subset $S$ of $Z$ if and only if

$$
x_{0} \leq s_{0} \leq y_{0}, \quad x_{1} \leq s_{1} \leq y_{1}
$$

for some liftings $s_{i} \in \tilde{Z}$ of the elements of $S$ (with the $x_{i}, y_{i}$ satisfying (2.1) above).

Proof. Choose liftings $x_{0}, x_{1} \in \tilde{Z}$ for the elements of $X$ so that $x_{0}<x_{1}<\sigma x_{0}$. If $\pi^{-1} Y$ has no elements in the half open interval $\left[x_{0}, x_{1}^{-}\right)$then any morphism $X \rightarrow Y$ in $\mathcal{M F}_{\phi}(Z)$ will factor through the projective-injective object $\pi\left\{x_{0}, x_{1}^{-}\right\}$. So, in order to have a nonzero morphism $X \rightarrow Y$ in $\mathcal{C}_{\phi}(Z)$, there must be a lifting $y_{0} \in\left[x_{0}, x_{1}^{-}\right)$of one of the elements of $Y$. Similarly, there must be a lifting $y_{1} \in\left[x_{1}, \sigma x_{0}^{-}\right)$of the other element of $Y$. Therefore, 2.1) is necessary to have a nonzero morphism $X \rightarrow Y$.

The statement about when a morphism $X \rightarrow Y$ factors through $S$ is clear and, assuming (2.1), $S$ cannot be projective-injective. Therefore $\mathcal{C}_{\phi}(Z)(X, Y) \neq 0$ when $(2.1)$ hold.

Lemma 2.4.3. In the triangulated category $\mathcal{C}_{\phi}(Z)$ we have $E\left(x_{0}, x_{1}\right)[1]=E\left(x_{1}^{-}, x_{0}^{-}\right)$. Furthermore, the shift functor [1] takes basic even morphisms to basic even morphisms and basic odd morphisms to negative basic odd morphisms. 
Proof. For any $x_{0} \neq x_{1}$ in $Z$ we choose the exact sequence in $\mathcal{M F}_{\phi}(Z)$ :

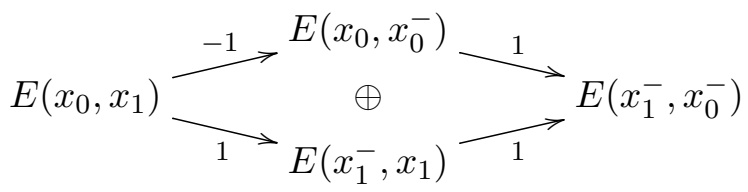

where the middle term is the injective envelope of $X=E\left(x_{0}, x_{1}\right)$ and the projective cover of $E\left(x_{1}^{-}, x_{0}^{-}\right)$. All four morphisms are even morphisms which are plus or minus a basic morphism as indicated. With this choice of injective envelopes for all indecomposable objects of $\mathcal{M F}_{\phi}(Z)$ we obtain $E\left(x_{1}^{-}, x_{0}^{-}\right)=E\left(x_{0}, x_{1}\right)[1]$ in the stable category $\mathcal{C}_{\phi}(Z)$. It is clear that this is natural with respect to even morphisms, i.e., that [1] takes basic even morphisms to basic even morphisms.

If we switch the order of $x_{0}, x_{1}$ then the signs on the two morphisms on the left will change. Therefore $\eta[1]=-\eta$ where $\eta: E\left(x_{0}, x_{1}\right) \cong E\left(x_{1}, x_{0}\right)$ is the basic odd isomorphism. This implies that [1] changes the sign of all odd morphisms.

Lemma 2.4.4. $\operatorname{Ext}^{1}(X, Y) \neq 0$ if and only if the subsets $X, Y$ of $Z$ are "crossing" in the sense that

$$
x_{0}<y_{0}<x_{1}<y_{1}<\sigma x_{0}
$$

for some liftings $x_{i}, y_{i} \in \tilde{Z}$ of the elements of $X, Y$. In particular, $\operatorname{Ext}^{1}(X, X)=0$.

Proof. This follows from the previous two lemmas.

Theorem 2.4.5. $\mathcal{C}_{\phi}(Z)$ is 2-Calabi-Yau, i.e., there is a natural isomorphism

$$
\operatorname{Ext}^{1}(X, Y) \cong D \operatorname{Ext}^{1}(Y, X)
$$

Proof. The lemma above implies that $\operatorname{Ext}^{1}(X, Y) \cong D E x t^{1}(Y, X)$ for any two indecomposable objects $X, Y$. It remains to show that the isomorphism is natural. Equivalently, we need to define a natural nondegenerate pairing

$$
\langle\cdot, \cdot\rangle: \operatorname{Ext}^{1}(X, Y) \otimes \operatorname{Ext}^{1}(Y, X) \rightarrow \mathbb{k}
$$

Since $\operatorname{Ext}^{1}(X, Y)=(X, Y[1])$ by definition, this pairing can be defined as the composition

$$
(X, Y[1]) \otimes(Y, X[1]) \stackrel{[1] \otimes i d}{\longrightarrow}(X[1], Y[2]) \otimes(Y, X[1]) \stackrel{\circ}{\rightarrow}(Y, Y[2]) \stackrel{\operatorname{Tr}}{\longrightarrow} \mathbb{k}
$$

where the trace map $\operatorname{Tr}:(Y, Y[2]) \rightarrow \mathbb{k}$ is given by choosing a decomposition of $Y$ into indecomposable objects $Y=\bigoplus Y_{i}$ and letting $\operatorname{Tr}(f)=\sum f_{i i}$ where $f_{i j} \in \mathbb{k}$ is the scalar corresponding to the $i j$ component of $f$ (since $\left(Y_{j}, Y_{i}\right)=\mathbb{k}$ or 0 ). Thus

$$
\langle f, g\rangle=\operatorname{Tr}(f[1] \circ g)
$$

It is clear that this pairing is natural in $X$. Naturality in $Y$ comes from the fact that [2] takes basic morphisms to basic morphisms (without changing sign) and thus $\operatorname{Tr}(g \circ h)=$ $\operatorname{Tr}(h[2] \circ g)$. Naturality in $Y$ also follows from the antisymmetry of the pairing:

$$
\langle f, g\rangle=-\langle g, f\rangle
$$

which follows from the fact that, for indecomposable $X$, any morphism $X \rightarrow X[2]$ must be odd. So, one of the morphisms $f$ or $g$ is odd and the other is even and [1], [-1] will reverse the sign of the odd morphism and preserve the sign of the even morphism making $\operatorname{Tr}(f[1] \circ g)=-\operatorname{Tr}(g[1] \circ f)$. 
2.4.2. Clusters. Clusters in $\mathcal{C}_{\phi}(Z)$ are maximal compatible sets of indecomposable objects which satisfy a certain continuity property which we now explain.

We recall that a Dedekind cut in $\tilde{Z}$ is a nonempty proper subset $S$ of $\tilde{Z}$ so that if $x<y$ and $y \in S$ then $x \in S$. We consider only proper Dedekind cuts, i.e., those which do not have suprema in $\tilde{Z}$. Note that $\sigma S \backslash S$ maps bijectively to $Z$ and induces a total ordering on the set $Z$ so that $Z$ does not have a maximum or minimum element. We call this a Dedekind ordering on $Z$. We say that a family of elements $z_{\alpha}$ in $Z$ converges to $\infty$ with respect to such an ordering if for all $x \in Z$ there exists $\alpha$ so that $z_{\alpha}>x$. Convergence to $-\infty$ is defined similarly.

Note that any nonempty subset $T$ of $Z$ either has a supremum or converges to $\infty$ with respect to another Dedekind ordering of $Z$. (Take the Dedekind cut $S$ consisting of all $z \in Z$ which are less than some element of $T$.)

For indecomposable $X \cong E(x, y)$, the points $x, y$ are called the endpoints of $X$.

Definition 2.4.6. A cluster if $\mathcal{C}_{\phi}(Z)$ is defined to be a maximal collection $\mathcal{T}$ of nonisomorphic indecomposable objects $T_{i}$ satisfying two properties:

(1) (Compatibility) $\operatorname{Ext}^{1}\left(T_{i}, T_{j}\right)=0$ for all $i, j$.

(2) (Limit Condition) Suppose that $T_{\alpha}$ is a transfinite sequence of objects in $\mathcal{T}$ so that one end $x_{\alpha}$ of $T_{\alpha}$ converges to $\infty$ with respect to some Dedekind ordering of $Z$ and the other end of $T_{\alpha}$ if fixed at, say $x$, then there is another transfinite sequence $S_{\beta}$ of objects in $\mathcal{T}$ so that one end of $S_{\beta}$ is fixed at the same point $x$ and the other endpoint of $S_{\beta}$ converges to $-\infty$ with respect to the same Dedekind ordering of $Z$.

We say that indecomposable $X, Y$ in $\mathcal{C}_{\phi}(Z)$ are compatible if $\operatorname{Ext}^{1}(X, Y)=0=\operatorname{Ext}^{1}(Y, X)$. Note that Limit Condition (2) is automatically satisfied if $\mathcal{T}$ is locally finite in the sense that, for every $x \in Z$, there are only finitely many objects in $\mathcal{T}$ with one end at $x$. In the case when $Z=\mathbb{Z}$, this definition is equivalent to the condition in [11] Theorem B and the proof that such sets satisfy the definition of a cluster is analogous. So, we omit the proof. However, we need to prove the symmetry of the above Limit Condition:

Lemma 2.4.7. The Limit Condition implies its converse, i.e., the same statement holds if $\infty$ and $-\infty$ are reversed.

Proof. Suppose that $T_{\alpha}$ is a collection of objects in $\mathcal{T}$ with one end fixed at $x$ and the other end converging to $-\infty$.

Claim $1 \mathcal{T}$ contains an object isomorphic to $E(x, y)$ for some $y>x$.

If not then, in particular, $E\left(x, x^{++}\right)$is not in $\mathcal{T}$ which is equivalent to saying that $\mathcal{T}$ has an object isomorphic to $E\left(x^{+}, y\right)$ where $y>x^{+}$. But the set of all such $y$ must have a supremum. Otherwise, by the Limit Condition, the $y$ 's must converge to $\infty$ which is impossible since $E\left(x^{+}, z\right)$ is not compatible with the objects $T_{\alpha}$ for any $z<x^{+}$. Let $y_{0}$ be this supremum. Then $E\left(x, y_{0}\right)$ is compatible with all objects in $\mathcal{T}$ and therefore an object in $\mathcal{T}$ (up to isomorphism). This proved Claim 1.

We want to show that the set of all $y>x$ so that $E(x, y)$ is in $\mathcal{T}$ (up to isomorphism) converges to $\infty$. If this is not the case then this set must have a supremum since, by the Limit Condition, if it converges to some Dedekind cut from below then it also converges to it from above. So, let $y_{1}$ be the supremum of this set. Then consider the set of all $z>y_{1}$ so that $\mathcal{T}$ contains an object isomorphic to $E\left(y_{1}, z\right)$. As in Claim 1, this set is nonempty and contains a maximal element $z_{1}$. But then $E\left(x, z_{1}\right)$ is compatible with all objects in $\mathcal{T}$ but is not contained in $\mathcal{T}$ since $z_{1}>y_{1}$. This contradicts the maximality of $\mathcal{T}$ and this contradiction proved the lemma. 
Lemma 2.4.8. $\mathcal{C}_{\phi}(Z)$ contains at least one cluster. Furthermore, for any object $T$ of any cluster $\mathcal{T}$, there is, up to isomorphism, a unique object $T^{*}$ so that $\mathcal{T} \backslash T \cup T^{*}$ is a cluster.

As in [11, the proof depends on the following.

Lemma 2.4.9. For any object $T=E(x, y)$ in any cluster $\mathcal{T}$ of $\mathcal{C}_{\phi}(Z)$, there are unique elements $a, b \in Z$ so that $\tilde{x}<\tilde{a}<\tilde{y}<\tilde{b}<\sigma \tilde{x}$ for some liftings $\tilde{x}, \tilde{y}, \tilde{a}, \tilde{b} \in \tilde{Z}$ of $x, y, a, b$ and so that $E(x, a), E(a, y), E(y, b), E(b, x)$ are either zero or isomorphic to objects in $\mathcal{T}$.

This implies that we have exact sequences $E(x, y) \rightarrow E(x, b) \oplus E(a, y) \rightarrow E(a, b)$ and $E(a, b) \rightarrow E(a, x) \oplus E(y, b) \rightarrow E(x, y)$ in the Frobenius category $\mathcal{M F}_{\phi}(Z)$ giving the following distinguished triangles in the triangulated category $\mathcal{C}_{\phi}(Z)$ :

$$
\begin{gathered}
T=E(x, y) \rightarrow E(x, b) \oplus E(a, y) \rightarrow T^{*}=E(a, b) \rightarrow T[1] \\
T[-1] \rightarrow T^{*}=E(a, b) \rightarrow E(a, x) \oplus E(y, b) \rightarrow T=E(x, y)
\end{gathered}
$$

where, up to isomorphism, $B=E(x, b) \oplus E(a, y)$ is a right $a d d\left(\mathcal{C}_{\phi}(Z) \backslash T\right)$-approximation of $T$ and $B^{\prime}=E(a, x) \oplus E(y, b)$ is a left $\operatorname{add}\left(\mathcal{C}_{\phi}(Z) \backslash T\right)$-approximation of $T$. As in [11] and as outlined below, this implies that $\mathcal{C}_{\phi}(Z)$ is a cluster category in the sense that it has a cluster structure according to the following definition from [3].

Definition 2.4.10. Suppose that $\mathcal{C}$ is a triangulated Krull-Schmidt category. Then a cluster structure on $\mathcal{C}$ is a collection of sets $\mathcal{T}$ called clusters of nonisomorphic indecomposable objects called variables satisfying the following conditions.

(a) For any cluster variable $T$ in any cluster $\mathcal{T}$ there is, up to isomorphism, a unique object $T^{*}$ not isomorphic to $T$ so that $\mathcal{T}^{*}:=\mathcal{T} \backslash T \cup T^{*}$ is a cluster.

(b) There are short exact sequences (or distringuished triangles)

$$
T^{*} \rightarrow B \rightarrow T, \quad T \rightarrow B^{\prime} \rightarrow T^{*}
$$

so that $B$ is a minimal right $\operatorname{add}(\mathcal{T} \backslash T)$-approximation of $T$ and $B^{\prime}$ is a minimal left $\operatorname{add}(\mathcal{T} \backslash T)$-approximation of $T$. We write $B=B_{\mathcal{T}}(T)$ and $B^{\prime}=B_{\mathcal{T}}^{\prime}(T)$.

(c) There are no loops or 2-cycles in the quiver of any cluster $\mathcal{T}$. (No loops means that any nonisomorphism $T \rightarrow T$ factors through $B_{\mathcal{T}}(T)$ and no 2-cycles means that there do not exist cluster variables $T, S$ in $\mathcal{T}$ so that $S$ is a summand of $B_{\mathcal{T}}(T)$ and $T$ is a summand of $B_{\mathcal{T}}(S)$.

(d) The quiver of $\mathcal{T}^{*}$ is obtained from the quiver of $\mathcal{T}$ by Fomin-Zelevinski mutation.

(e) If $\mathcal{T}^{\prime}$ is obtained from $\mathcal{T}$ by replacing each variable with an isomorphic object then $\mathcal{T}^{\prime}$ is a cluster.

Proof of Theorem 2.4.1. We have already shown conditions (a) and (b) and condition (e) holds by definition. Condition (c) is easy since the only arrows in the quivers of $\mathcal{T}$ starting or ending at $T=E(x, y)$ and the only arrow in the quiver of $\mathcal{T} \backslash T \cup T^{*}$ starting or ending at $T^{*}=E(a, b)$ are given in the following diagram.
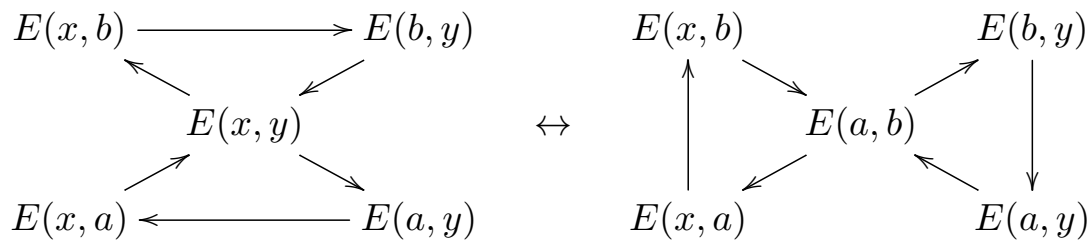
Some of the terms may be zero and should be deleted. Condition (d) holds by examination of the above diagram. Therefore, $\mathcal{C}_{\phi}(Z)$ has a cluster structure. By Theorem 2.4.5, it is 2-Calabi-Yau.

2.4.3. Example. We discuss explicit examples of discrete cluster categories.

Lemma 2.4.11. $\mathcal{C}_{\phi}(Z)$ has almost split triangles given by

$$
E(x, y) \rightarrow E\left(x, y^{+}\right) \oplus E\left(x^{+}, y\right) \rightarrow E\left(x^{+}, y^{+}\right) \rightarrow E\left(y^{-}, x^{-}\right)
$$

where $y \neq x^{++}$and

$$
E\left(x^{-}, x^{+}\right) \rightarrow E\left(x^{-}, x^{++}\right) \rightarrow E\left(x, x^{++}\right) \rightarrow E\left(x, x^{--}\right)
$$

Up to isomorphism, the irreducible maps are the basic morphisms $E(x, y) \rightarrow E\left(x, y^{+}\right)$.

Proof. The fact that the first sequence is a distinguished triangle (up to the signs of the morphisms) follows from that fact that $E(x, y) \rightarrow E\left(x, y^{+}\right) \oplus E\left(x^{+}, y\right) \rightarrow E\left(x^{+}, y^{+}\right)$is an exact sequence in the Frobenius category. The second sequence is an example of a "positive triangle" [14. This is a distinguished triangle where all three morphisms are basic.

It is easy to see that these are almost split triangles: Given any morphism $f: E(a, b) \rightarrow$ $E\left(x^{+}, y^{+}\right)$which is not an isomorphism, assuming that $f$ is diagonal, either $a \neq x^{+}$in which case $f$ factors through $E\left(x, y^{+}\right)$or $b \neq y$ in which case $f$ factors through $E\left(x^{+}, y\right)$. Similarly, any nonisomorphism $E(x, y) \rightarrow E(a, b)$ factors through $E\left(x, y^{+}\right) \oplus E\left(x^{+}, y\right)$. So, the first triangle is almost split. A similar argument shows that the second triangle is almost split. The description of the irreducible morphisms is clear since $x \rightarrow x^{+}$are the irreducible morphisms in $\mathcal{P}(Z)$.

Lemma 2.4.12. Let $(Z, \phi)$ be as in Theorem 2.4.1. Then either $Z \cong Z_{n}$ with $\phi(i)=i+1$ or $Z \cong S * \mathbb{Z}$ where $S$ is cyclically ordered and $\phi(s, i)=(s, i+1)$.

Proof. We prove only the infinite case. The automorphism $\phi$ gives an action of the additive group $(\mathbb{Z},+)$ on the set $Z$. When $Z$ is infinite, this must be a free action. Let $S$ be the set of orbits of this action. Then $S$ is cyclically ordered and $Z \cong S * \mathbb{Z}$.

Theorem 2.4.13. The Auslander-Reiten quiver of $\mathcal{C}_{\phi}(Z) \cong \mathcal{C}_{\phi}(S * \mathbb{Z})$ is a union of $\mathbb{Z} A_{\infty}$ components $C_{s}$ indexed by the elements of $s \in S$ and $\mathbb{Z} A_{\infty}^{\infty}$ components $C_{a b}$ indexed by unordered pairs of distinct elements of $S$.

Proof. For every $s \in S$, the $C_{s}$ component consists of all objects $E((s, i),(s, j))$ where $i, j \in \mathbb{Z}$ with $|i-j| \geq 2$. Since these contain the simple objects $E((s, i),(s, i+2))$, they are of type $\mathbb{Z} A_{\infty}$. For $a \neq b \in S$, the component $C_{a b}$ contains all objects $E((a, i),(b, j))$ where $i, j \in \mathbb{Z}$. This is a component of type $\mathbb{Z} A_{\infty}^{\infty}$.

An example of a cluster in $\mathcal{C}_{\phi}(Z)$ for $S=\mathbb{Z}\left(Z=\mathbb{Z}^{2}\right.$ with lexicographic order $)$ is given by taking a "zig-zag" in each component $C_{i, i+1}$ starting at the center point $E((i, 0),(i+$ $1,0))$ plus a zig-zag pattern of center points. By a zig-zag in $C_{a b}$ we mean a sequence of objects $X_{0}, X_{1}, \cdots$ so that, if $X_{k}=E((a, i),(b, j))$ then $X_{k+1}=E((a, i+1),(b, j))$ or $E((a, i),(b, j-1))$ and so that $i \rightarrow \infty$ and $j \rightarrow-\infty$ as $k \rightarrow \infty$. By a zig-zag pattern of center points we mean a sequence of objects $Y_{0}, Y_{1}, \cdots$ where each $Y_{k}=E((i, 0),(j, 0))$ and $Y_{k+1}=E((i-1,0),(j, 0))$ or $E((i, 0),(j+1,0))$ and so that $Y_{0}=E((i, 0),(i+1,0))$ for some $i$ and so that $i \rightarrow-\infty$ and $j \rightarrow \infty$ as $k \rightarrow \infty$. See Figure 1 . 


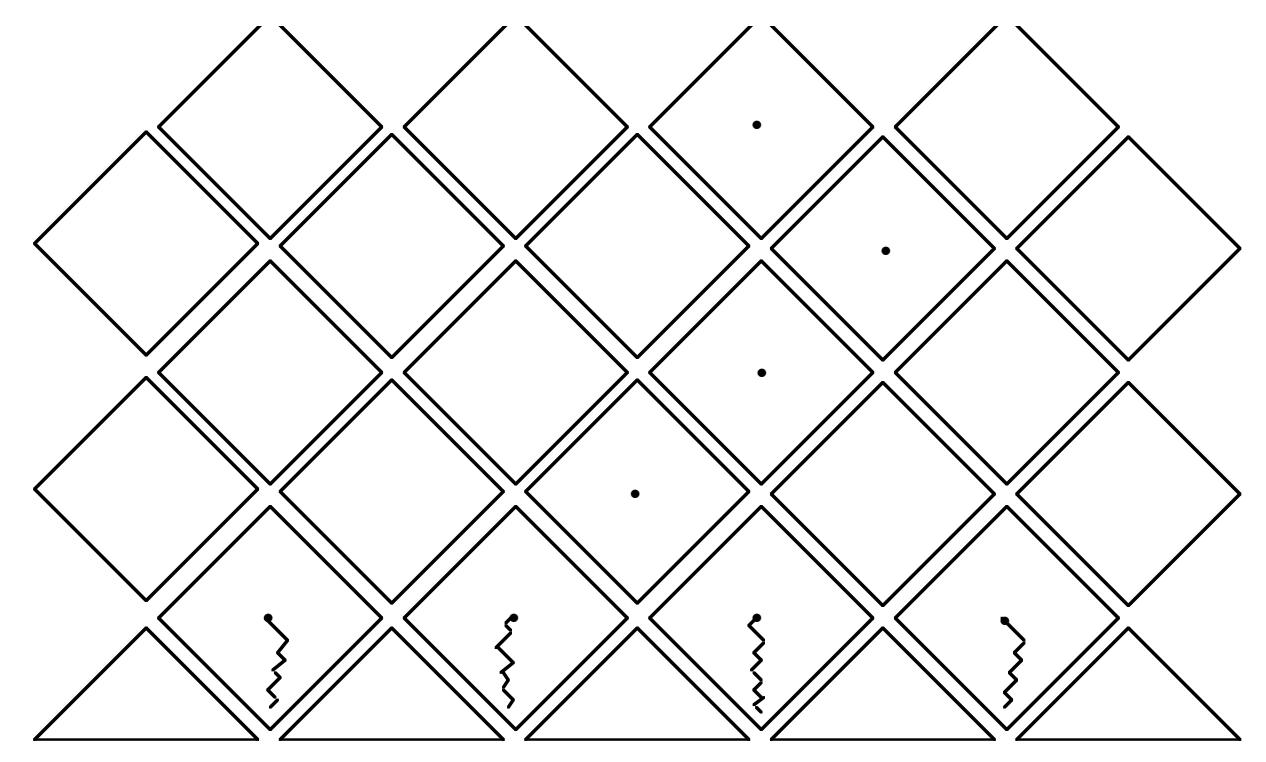

Figure 1. An example of a cluster in $\mathcal{C}_{\phi}\left(\mathbb{Z}^{2}\right)$. In each $C_{i, i+1}$ component, choose a zig-zag starting at the center point $((0,0)$ followed by an infinite sequence of objects given by adding $(1,0)$ or $(0,-1)$ at each step and converging to $(\infty,-\infty))$. Then take the center points of $C_{i j}$ where $(i, j)$ form another zig-zag patter, such as $(0,2),(0,3),(0,4),(-1,4), \cdots$ (adding $(0,1)$ or $(-1,0)$ at each step and converging to $(-\infty, \infty))$.

2.5. $m$-cluster category of type $A_{\infty}$. As another example, we will construct a triangulated category $\mathcal{C}_{\Phi}\left(Z_{m} * \mathbb{Z}\right)$ whose standard objects form a thick subcategory $\mathcal{C}_{\infty}^{m}$ which satisfies the definition of an $m$-cluster category for $m \geq 3$. We will show that the standard $m$-clusters are in bijection with the set of partitions of the $\infty$-gon into $m+2$-gons. Such a category has already been constructed in [12. We also show that the triangulated category $\mathcal{C}_{\Phi}\left(Z_{m} * \mathbb{Z}\right)$ is $m+1$-Calabi-Yau and that its $m+1$-rigid objects have a partial cluster structure. We call maximal compatible sets of $m+1$-rigid objects nonstandard clusters. These correspond to 2-periodic partitions of the double $\infty$-gon into $m+2$-gons plus one $m+3$-gon in the middle. The sides of this middle polygon have more than $m$ mutations. These structures can also be obtained by taking $m$-cluster categories of type $A_{n}$ as in [1] and taking the limit as $n$ goes to $\infty$.

2.5.1. Definitions. Let $m \geq 3$ and let $Z_{\infty}^{m}=Z_{m} * \mathbb{Z}$ with elements $(p, k)$ denoted by $x_{k}^{p}$ where $p=1, \cdots, m, k \in \mathbb{Z}$. Let $\Phi: Z_{\infty}^{m} \rightarrow Z_{\infty}^{m}$ be the automorphism given by

$$
\Phi\left(x_{k}^{p}\right)= \begin{cases}x_{k}^{p+1} & \text { if } p \neq m \\ x_{k+1}^{1} & \text { if } p=m\end{cases}
$$

Then $\Phi^{m}\left(x_{k}^{p}\right)=x_{k+1}^{p}$ which is the successor of $x_{k}^{p}$ for any $x_{k}^{p} \in Z_{\infty}^{m}$. Since $x, \Phi(x), \Phi^{2}(x)$ are in cyclic order, $\Phi$ is an admissible automorphism of $Z_{\infty}^{m}$. To simplify notation we use the convention that $x_{k}^{p+m}=x_{k+1}^{p}$ for all integers $p, k$ and, more generally, $x_{k}^{p}=x_{j}^{q}$ if $m k+p=m j+q$. In other words, $\lambda\left(x_{k}^{p}\right)=m k+p$ gives a bijection $\lambda: Z_{\infty}^{m} \rightarrow \mathbb{Z}$. For example, the formula for $\Phi$ is $\Phi\left(x_{k}^{p}\right)=x_{k}^{p+1}$ for all integers $k, m$. Note that $\lambda$ does not preserve order. 
It is important to observe that $\Phi^{m}\left(x_{k}^{p}\right)=x_{k}^{p+m}=x_{k+1}^{p}$ is the successor of $x_{k}^{p}$.

By Theorem 2.1.7 we have:

Proposition 2.5.1. $\mathcal{M F}_{\Phi}\left(Z_{\infty}^{m}\right)$ is a Frobenius category with indecomposable objects $E(x, y)$ where $x, y \in Z_{\infty}^{m}$ so that $\left(\Phi(x), y, \Phi^{-1}(x)\right)$ is in cyclic order, i.e., either $\Phi^{-1}(x)<\Phi(x) \leq y$, $y \leq \Phi^{-1}(x)<\Phi(x)$ or $\Phi(x) \leq y \leq \Phi^{-1}(x)$. Also, we have:

$$
E\left(x_{i}^{p}, x_{j}^{q}\right)[1]=E\left(\Phi^{-1} x_{j}^{q}, \Phi^{-1} x_{i}^{p}\right)=E\left(x_{j}^{q-1}, x_{i}^{p-1}\right)
$$

The shift operator [1] takes basic even morphism to basic even morphisms and basic odd morphisms to -1 times basic odd morphisms.

Since $\Phi^{-m}\left(x_{i}^{p}\right)=x_{i}^{p-m}=x_{i-1}^{p}$ is the predecessor of $x_{i}^{p}$, we conclude that

$$
E(x, y)[m]=E\left(x^{-}, y^{-}\right)
$$

Lemma 2.4.11 still holds, except that the formula for the shift [1] has changed. The new lemma is:

Lemma 2.5.2. The stable category $\underline{\mathcal{M F}} \mathcal{F}_{\Phi}\left(Z_{\infty}^{m}\right)=\mathcal{C}_{\Phi}\left(Z_{\infty}^{m}\right)$ has almost split triangles

$$
\tau E(x, y) \rightarrow E\left(x^{-}, y\right) \oplus E\left(x, y^{-}\right) \rightarrow E(x, y) \rightarrow \tau E(x, y)[1]
$$

where $\tau E(x, y)=E\left(x^{-}, y^{-}\right) \cong E(x, y)[m]$.

Theorem 2.5.3. The triangulated category $\mathcal{C}_{\Phi}\left(Z_{\infty}^{m}\right)$ is $m+1$-Calabi-Yau, i.e., there is a natural isomorphism

$$
\operatorname{Ext}^{k}(X, Y) \cong D \operatorname{Ext}^{m+1-k}(Y, X)
$$

for all objects $X, Y$.

Proof. The lemma implies that the basic morphism $E(x, y) \rightarrow \tau E(x, y)[1]=E(x, y)[m+1]$ is a hammock which means that for any morphism $E(x, y) \rightarrow Y$ for any object $Y$, there is a morphism $Y \rightarrow E(x, y)[m+1]$. The duality is given by the fact that the nondegenerate pairing

$$
(X, Y) \otimes(Y, X[m+1]) \rightarrow(X, X[m+1]) \cong \mathbb{k}
$$

As in the proof of Theorem 2.4.5, naturality of the pairing follows from the fact that it is symmetric up to sign.

2.5.2. Auslander-Reiten quiver of $\mathcal{C}_{\Phi}\left(Z_{\infty}^{m}\right)$. As in the previous section, we have the following.

Theorem 2.5.4. The Auslander-Reiten quiver of $\mathcal{C}_{\Phi}\left(Z_{\infty}^{m}\right)$ is a union of $\left(\begin{array}{c}m \\ 2\end{array}\right)$ components $C_{p q}=C_{q p}$ where $p, q$ are distinct integers modulo $m$. Of these, the $m$ components $C_{p, p+1}$ are of type $\mathbb{Z} A_{\infty}$ and the others are of type $\mathbb{Z} A_{\infty}^{\infty}$.

Proof. The points in $Z_{m} * \mathbb{Z}$ form $m$ blocks given by fixing the first coordinate. Objects $E(x, y)$ must have ends $x, y$ in different blocks. We let $C_{p q}$ be the set of all $E\left(x_{i}^{p}, x_{j}^{q}\right)$. These form a component of the Auslander-Reiten quiver since irreducible maps will change the subscripts by 1 . If $q=p+1$ then we must have $j>i$. So, $C_{p, p+1}$ is of type $\mathbb{Z} A_{\infty}$. The others have type $\mathbb{Z} A_{\infty}^{\infty}$ since $i, j$ are arbitrary. 


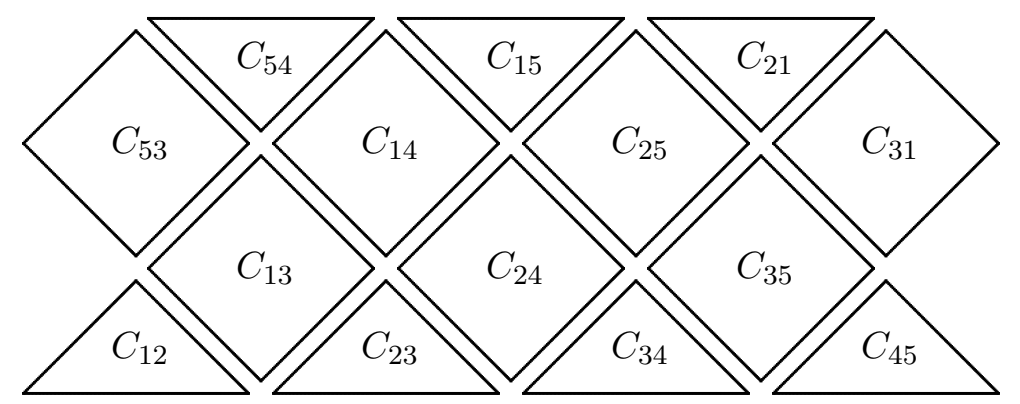

Figure 2. When $m=5$, there are $\left(\begin{array}{c}m \\ 2\end{array}\right)=10$ components in the AR-quiver of $\mathcal{C}_{\Phi}\left(Z_{\infty}^{m}\right)$. They are labeled $C_{p q}=C_{q p}$. When $p, q$ are consecutive, $C_{p q}$ has type $\mathbb{Z} A_{\infty}$. Otherwise $C_{p q}$ has type $\mathbb{Z} A_{\infty}^{\infty}$. Four components are repeated.

2.5.3. Standard objects and $m$-clusters. We define the standard objects of $\mathcal{C}_{\Phi}\left(Z_{\infty}^{m}\right)$ to be those objects all of whose indecomposable summands lie in the $\mathbb{Z} A_{\infty}$ components $C_{p, p+1}$ of the Auslander-Reiten quiver of $\mathcal{C}_{\Phi}\left(Z_{\infty}^{m}\right)$. In other words, the standard indecomposable objects are $E\left(x_{i}^{p}, x_{j}^{p+1}\right)$ where $i<j$.

The main properties of standard objects are given below. We recall that an indecomposable object $X$ in an $m+1$-Calabi-Yau triangulated category if $m+1$-rigid if $\operatorname{Ext}^{k}(X, X)=0$ for $1 \leq k \leq m$. Two such objects are compatible if $\operatorname{Ext}^{k}(X, Y)=0$ for $1 \leq k \leq m$.

Theorem 2.5.5. The full subcategory of $\mathcal{C}_{\Phi}\left(Z_{\infty}^{m}\right)$ consisting of standard objects is a thick subcategory. This subcategory is a true $m$-cluster category in the following sense.

(1) All standard indecomposable objects $X$ are $m+1$-rigid.

(2) Define a standard $m$-cluster to be a maximal compatible set of indecomposable standard objects of $\mathcal{C}_{\Phi}\left(Z_{\infty}^{m}\right)$. Then for any such set $\mathcal{T}$ and any object $T \in \mathcal{T}$, there are exactly $m$ other standard objects $T_{i}^{*}$ up to isomorphism so that $\mathcal{T} \backslash T \cup T_{i}^{*}$ is a standard $m$-cluster.

(3) The objects $T_{i}^{*}$ with $T_{0}^{*}=T$ are uniquely determined up to isomorphism by the fact that there is a distinquished triangle

$$
T_{i}^{*} \rightarrow B_{i} \rightarrow T_{i+1}^{*} \rightarrow T_{i}^{*}[1]
$$

where $B_{i}$ is the left add $\mathcal{T} \backslash T$-approximation of $T_{i}^{*}$.

Furthermore, the Verdier quotient $\mathcal{C}\left(Z_{\infty}^{m}\right) / \mathcal{C}_{\infty}^{m}$ is triangle equivalent to the standard cluster category of type $A_{m-3}$.

If $X=E(x, y)$ then let $\lambda_{1} X<\lambda_{2} X$ be the integers $\lambda(x), \lambda(y)$ in increasing order and let $\lambda X=\left(\lambda_{1} X, \lambda_{2} X\right)$. We say that $X$ crosses $Y$ if $\lambda_{1} X<\lambda_{1} Y<\lambda_{2} X<\lambda_{2} Y$.

Lemma 2.5.6. Two standard objects $X, Y$ are compatible if and only if they are noncrossing, i.e., they do not cross each other.

Since $X$ never crosses itself, this implies that standard objects are $m+1$-rigid. Lemma 2.5.6 also implies the following theorem.

Theorem 2.5.7. Isomorphism classes of standard $m$-clusters are in 1-1 correspondence with the partitions of the $\infty$-gon into $m+2$-gons.

Definition 2.5.8. By the $\infty$-gon we mean the convex hull $P_{\infty}$ of the set $V$ of all points on the unit circle in $\mathbb{C}$ of the form $e^{i \theta}$ where $\cot \frac{\theta}{2} \in \mathbb{Z}$. This gives a bijection $V \cong \mathbb{Z}$. We call 
$V$ the vertex set of $P_{\infty}$. By a partition of $P_{\infty}$ we mean a set of noncrossing chords in the circle with both endpoints in the vertex set $V$. (See [11].)

Proof. For any standard object $X=E(x, y)$, take the chord in the $\infty$-gon with endpoints corresponding to $\lambda(x), \lambda(y) \in \mathbb{Z}$. If $X, Y$ are compatible then the corresponding chords do not cross. Therefore, any standard $m$-cluster gives a partition of the $\infty$-gon $P_{\infty}$ into polygons. Take one of these polygons, say $P$ and let $(a, b)=\lambda X$ be the longest side of $P$. Since $X$ is standard, $b-a \equiv 1 \bmod m$ and $b \geq a+m+1$. If $b=a+m+1$ then the other sides of $P$ are sides of $P_{\infty}$ and $P$ is an $m+2$-gon. Otherwise, take the shortest chord $C=(v, w)$ inside the closed interval $[a, b]$. Then $C$ has length $m+1$ and bounds an $m+2$-gon. Contract $C$ to the side $(v, v+1)$ on $P_{\infty}$ and subtract $m$ from the position of all vertices $\geq w$. Then $b-a$ decreases by $m$ and we conclude by induction on $b-a$ that $P$ is an $m+2$-gon. The converse argument is similar.

To prove Lemma 2.5.6, we examine which standard objects map to each other.

Lemma 2.5.9. Let $X, Y$ be two standard objects in the same component of the AR-quiver. Then $\mathcal{C}_{\Phi}(X, Y) \neq 0$ if and only if

$$
\lambda_{1} X \leq \lambda_{1} Y<\lambda_{2} X-1 \leq \lambda_{2} Y-1
$$

The proof is straightforward and left to the reader. Note that these four integers are congruent to each other modulo $m$.

Lemma 2.5.10. Let $X, Y$ be standard objects. Then the following two statements are equivalent.

(1) There exists $1 \leq k \leq m$ so that $Y[k]$ lies in the same $A R$-component as $X$ and so that $\mathcal{C}_{\Phi}(X, Y[k]) \neq 0$.

(2) $X$ crosses $Y$, i.e., $\lambda_{1} X<\lambda_{1} Y<\lambda_{2} X<\lambda_{2} Y$.

Proof. Without loss of generality we may assume that $\lambda_{1} X$ is divisible by $m$. Thus, when $Y[k]$ lies in the same AR-component as $X, \lambda_{1} Y[k]$ will also be divisible by $m$ and $\lambda_{1} Y[k] / m=\left\lfloor\left(\lambda_{1} Y-1\right) / m\right\rfloor$. Similarly, $\lambda_{2} Y[k]-1$ is divisible by $m$ and $\left(\lambda_{2} Y[k]-1\right) / m=$ $\left\lfloor\left(\lambda_{2} Y-2\right) / m\right\rfloor$. So, by (2.2), Condition (1) is equivalent to the integer inequality:

$$
\frac{\lambda_{1} X}{m} \leq\left\lfloor\frac{\lambda_{1} Y-1}{m}\right\rfloor<\frac{\lambda_{2} X-1}{m} \leq\left\lfloor\frac{\lambda_{2} Y-2}{m}\right\rfloor
$$

For all integers $a, b$ and real numbers $x, y$, the integer inequality $a \leq\lfloor x\rfloor<b \leq\lfloor y\rfloor$ is equivalent to the real inequality $a \leq x<b \leq y$. So, 2.3) is equivalent to the condition

$$
\lambda_{1} X \leq \lambda_{1} Y-1<\lambda_{2} X-1 \leq \lambda_{2} Y-2
$$

which is equivalent to Condition (2).

Lemma 2.5.11. Let $X, Y$ be standard objects. Then the following two statements are equivalent.

(1) There exists $1 \leq k \leq m$ so that $\mathcal{C}_{\Phi}(X, Y[k]) \neq 0$ but $Y[k]$ does not lie in the same AR-component as $X$.

(2) $Y$ crosses $X$, i.e., $\lambda_{1} Y<\lambda_{1} X<\lambda_{2} Y<\lambda_{2} X$.

Proof. Since $\mathcal{C}_{\Phi}$ is $m+1-\mathrm{CY}$, the condition $\mathcal{C}_{\Phi}(X, Y[k]) \neq 0$ is equivalent to the condition that $\mathcal{C}_{\Phi}(Y, X[m+1-k]) \neq 0$. In Condition (1) we must also have $\lambda_{1} Y[k]=\lambda_{1} Y-k \equiv \lambda_{1} X-1$ modulo $m$. So, $\lambda_{1} X[m+1-k] \equiv \lambda_{1} X-1+k \equiv \lambda_{1} Y \bmod m$. Thus, Condition (1) is 
equivalent to the condition that there exists $1 \leq \ell \leq m$ so that $X[\ell]$ lies in the same AR-component as $Y$ and $\mathcal{C}_{\Phi}(Y, X[\ell]) \neq 0$. By the previous lemma, this is equivalent to Condition (2).

Proof of Lemma 2.5.6. There are two ways that $X, Y$ might be not compatible. By Lemmas 2.5 .10 and 2.5.11 these correspond exactly to the two different ways that $X, Y$ can cross. Therefore, they are compatible iff they don't cross.

Lemma 2.5.12. Suppose $X, Y$ are nonisomorphic compatible standard objects in $\mathcal{C}_{\Phi}\left(Z_{\infty}^{m}\right)$. Then $\mathcal{C}_{\Phi}(X, Y) \neq 0$ if and only if one of the following holds.

(1) $\lambda_{1} X=\lambda_{1} Y$ and $\lambda_{2} X<\lambda_{2} Y$.

(2) $\lambda_{1} X<\lambda_{1} Y$ and $\lambda_{2} X=\lambda_{2} Y$.

(3) $\lambda_{1} X=\lambda_{2} Y$.

Proof. If $X, Y$ are in the same AR-component, $\mathcal{C}_{\Phi}(X, Y) \neq 0$ is equivalent to (1) or (2) by Lemma 2.5.9 given that $X, Y$ are compatible and thus noncrossing. If $X, Y$ are not in the same AR-component then $\mathcal{C}_{\Phi}(X, Y) \neq 0$ is equivalent to $\mathcal{C}_{\Phi}(Y, X[m+1]) \neq 0$ with $Y, X[m+1]$ being in the same AR-component. By Lemma 2.5.9 this is equivalent to:

$$
\lambda_{1} Y \leq \lambda_{1} X-m-1<\lambda_{2} Y-1 \leq \lambda_{2} X-m-2 .
$$

Since these integers are congruent to each other modulo $m$, this is equivalent to:

$$
\lambda_{1} Y<\lambda_{1} X-1 \leq \lambda_{2} Y-1<\lambda_{2} X-2,
$$

a condition equivalent to (3) given that $X, Y$ are noncrossing.

Proof of Theorem 2.5.5. We are now ready to prove the main theorem about standard $m$ clusters.

(1) We have already observed that standard objects are $m+1$-rigid since they do not cross themselves.

(2) Every element $T$ of every standard $m$-cluster $\mathcal{T}$ represents a chord in $P_{\infty}$ which separates two $m+2$-gons. When the chord is removed, we have a $2 m+2$-gon $G$ which has $m+1$ diagonals. So, there are $m$ other diagonals corresponding to $m$ other standard objects $T_{i}^{*}$ so that $\mathcal{T} \backslash T \cup T_{i}^{*}$ forms a standard $m$-cluster.

(3) By Lemma 2.5.12, morphisms between compatible standard objects correspond to counterclockwise pivots of the corresponding chords in $P_{\infty}$. So, the left add $\mathcal{T} \backslash T$-approximation of $T_{i}^{*}$ is given by the direct sum of the two objects $B_{i}^{\prime}, B_{i}^{\prime \prime}$ (corresponding to chords) which form the sides of the $2 m+2$-gon $G$ which are adjacent to $T_{i}^{*}$ and are clockwise from the endpoints of $T_{i}^{*}$ as shown in the figure. The sequence

$$
T_{i}^{*} \stackrel{\left[\begin{array}{c}
1 \\
-1
\end{array}\right]}{\longrightarrow} B_{i}^{\prime} \oplus B_{i}^{\prime \prime} \stackrel{[1,1]}{\longrightarrow} T_{i+1}^{*}
$$

is exact in $\mathcal{M F}_{\Phi}\left(Z_{\infty}^{m}\right)$ and therefore forms a distinguished triangle in the stable category $\mathcal{C}_{\Phi}\left(Z_{\infty}^{m}\right)$. Thus the objects $T_{i}^{*}$ form the diagonals of the $2 m+2$-gon $G$ ordered clockwise.

To show that the standard objects form a thick subcategory $\mathcal{C}_{\infty}^{m}$ of $\mathcal{C}_{\Phi}\left(Z_{\infty}^{m}\right)$, it suffices to show that $\mathcal{C}_{\infty}^{m}$ is a triangulated full subcategory, in other words, for any morphism of standard objects $f: X \rightarrow Y$, the third object in the distinguished triangle $X \rightarrow Y \rightarrow Z \rightarrow$ $X[1]$ is also standard. By the octahedral axiom it suffices to consider the case when $X$ is indecomposable. So, suppose that $X \in C_{p, p+1}$. Then, dropping all of the components of the map $f: X \rightarrow Y$ which are zero, we may assume that $Y$ lies in $C_{p, p+1} \cup C_{p-1, p}$. Since $X[1]$ lies 


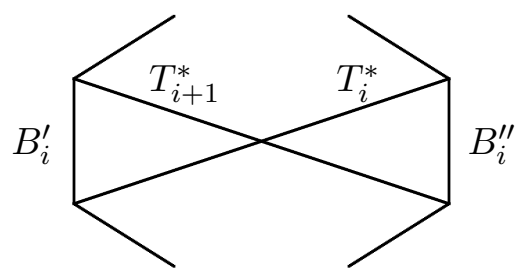

Figure 3 . The objects $B_{i}^{\prime}$ and $B_{i}^{\prime \prime}$ share an endpoint with $T_{i}^{*}$ and are the objects in $\mathcal{T} \backslash T$ closest to $T_{i}^{*}$ in the counterclockwise direction. So, their sum $B_{i}=B_{i}^{\prime} \oplus B_{i}^{\prime \prime}$ form the left $\mathcal{T} \backslash T$-approximation to $T_{i}^{*}$ and $T_{i}^{*} \rightarrow B_{i}^{\prime} \oplus B_{i}^{\prime \prime} \rightarrow$ $T_{i+1}^{*}$ is a distinguished triangle in $\mathcal{C}_{\Phi}\left(Z_{\infty}^{m}\right)$.

in $C_{p-1, p}$, all of the endpoints of the object $Z$ must lie in blocks $p-1, p, p+1$. Therefore, the only possible nonstandard components of $Z$ lie in $C_{p-1, p}$. But this is impossible since none of these objects map nontrivially to $X[1] \in C_{p-1, p}$. So, all components of $Z$ are standard.

Finally, to identify the Verdier quotient category $\mathcal{C}_{\Phi}\left(Z_{\infty}^{m}\right) / \mathcal{C}_{\infty}^{m}$, we claim that the objects of this quotient are the components of the AR-quiver of $\mathcal{C}_{\Phi}\left(Z_{\infty}^{m}\right)$. This follows from two considerations:

(1) Any two indecomposable objects in the same component $C_{p q}$ are equivalent in $\mathcal{C}_{\Phi}\left(Z_{\infty}^{m}\right) / \mathcal{C}_{\infty}^{m}$

(2) There is an exact morphism of Frobenius categories $\mathcal{M F}_{\Phi}\left(Z_{\infty}^{m}\right) \rightarrow \mathcal{M F}_{\phi}\left(Z_{m}\right)$ so that the inverse image of the indecomposable objects of $\mathcal{M F}_{\phi}\left(Z_{m}\right)$ are subcategories of $\mathcal{M F}_{\Phi}\left(Z_{\infty}^{m}\right)$ which map onto the AR-components of $\mathcal{C}_{\Phi}\left(Z_{\infty}^{m}\right)$ and the inverse image of the projective-injective objects are exactly the standard objects (and the projective-injective objects) of $\mathcal{M F}_{\Phi}\left(Z_{\infty}^{m}\right)$.

The first statement is easy to see. For example, there is an exact sequence

$$
E\left(x_{i}^{p}, x_{j}^{q}\right) \rightarrow E\left(x_{k}^{p}, x_{j}^{q}\right) \oplus E\left(x_{i}^{p}, x_{N}^{p+1}\right) \rightarrow E\left(x_{k}^{p}, x_{N}^{p+1}\right)
$$

for sufficiently large $N$ showing that $E\left(x_{i}^{p}, x_{j}^{q}\right) \sim E\left(x_{k}^{p}, x_{j}^{q}\right)$ modulo standard objects and, similarly, $E\left(x_{i}^{p}, x_{j}^{q}\right) \sim E\left(x_{i}^{p}, x_{k}^{q}\right)$.

The exact morphism in the second statement is induced by the morphism of cyclic posets $p: Z_{m} * \mathbb{Z} \rightarrow Z_{m}$ given by projection to the first coordinate. This morphism is compatible with $\Phi$ and $\phi$ since $\phi \circ p=p \circ \Phi$. Therefore, it induces an exact functor $\mathcal{M} \mathcal{F}_{\Phi}\left(Z_{\infty}^{m}\right) \rightarrow \mathcal{M} \mathcal{F}_{\phi}\left(Z_{m}\right)$ sending projective-injective objects to projective injective objects and it is easy to see that it has the stated properties.

We saw that the endpoints of a standard object lie in two consecutive blocks: $x_{*}^{p}$ and $x_{*}^{p+1}$. We now consider nonstandard objects (with blocks a distance of at least 2 apart).

\subsubsection{Nonstandard objects and periodic m-clusters.}

Proposition 2.5.13. An indecomposable object $X$ of $\mathcal{C}_{\Phi}\left(Z_{\infty}^{m}\right)$ is $m+1$-rigid iff it is one of the following.

(1) $X$ is standard.

(2) $X=E\left(x_{i}^{p}, x_{j}^{p+2}\right)$ where $i>j$. 
Remark 2.5.14. We call objects of the second kind nonstandard $m+1$-rigid objects. Since $\lambda\left(x_{i}^{p}\right)>\lambda\left(x_{j}^{p+2}\right)$, the nonstandard $m+1$ rigid objects can also be described as those objects $Y$ where $\lambda_{1} Y-\lambda_{2} Y \equiv 2 \bmod m$.

Proof. Suppose first that the endpoints of $X$ lie in blocks at least 3 apart: $X=E\left(x_{i}^{p}, x_{j}^{q}\right)$ where $p+3 \leq q \leq p+m-3$. Then $X[m-1]=E\left(x_{i-1}^{p+1}, x_{j-1}^{q+1}\right)$ and $\mathcal{C}_{\Phi}(X, X[m-1]) \neq 0$. So, $X$ is not rigid. Next, suppose that $X=E\left(x_{i}^{p}, x_{j}^{p+2}\right)$. Then the only possible self-extension of $X$ is $\mathcal{C}_{\Phi}(X, X[m-1])$. Since $X[m-1]=E\left(x_{i-1}^{p+1}, x_{j-1}^{p+3}\right)$ we have $\mathcal{C}_{\Phi}(X, X[m-1]) \neq 0$ iff $i-1<j$. So we need $j<i$ for $X=E\left(x_{i}^{p}, x_{j}^{p+2}\right)$ to be rigid.

Corollary 2.5.15. All objects of $\mathcal{C}_{\Phi}\left(Z_{\infty}^{m}\right)$ are $m+1$ rigid iff $m \leq 4$.

Define a nonstandard $m$-cluster to be a maximal collection of pairwise compatible $m+1$ rigid indecomposable objects of $\mathcal{C}_{\Phi}\left(Z_{\infty}^{m}\right)$ containing at least one nonstandard object.

Let $B=\mathbb{R} \times[0,1]$ and let $\partial B^{(2)}$ be the set of all two element subsets of the boundary $\partial B=\mathbb{R} \times\{0,1\}$. Let $\lambda_{0}, \lambda_{1}: Z_{\infty}^{m} \rightarrow \partial B^{(2)}$ be the two embeddings given by $\lambda_{0} x=(\lambda(x), 0)$, $\lambda_{1} x=(-\lambda(x), 1)$.

Theorem 2.5.16. Let $\Psi$ be the mapping which sends any standard object $E(x, y)$ to the pair of pairs $\Psi E(x, y)=\left\{\left(\lambda_{0} x, \lambda_{0} y\right),\left(\lambda_{1} x, \lambda_{1} y\right)\right\}$ and any nonstandard $m+1$-rigid object $E(x, z)$ to $\Psi E(x, z)=\left\{\left(\lambda_{0} x, \lambda_{1} z\right),\left(\lambda_{1} x, \lambda_{0} z\right)\right\}$. Then a collection of rigid indecomposable objects of $\mathcal{C}_{\Phi}\left(Z_{\infty}^{m}\right)$ form a nonstandard $m$-cluster iff it is mapped by $\Psi$ to a 2-periodic decomposition of the $A_{\infty}^{2}$-gon into $m+2$-gons (except for the one in the middle).

The proof follows the same pattern as the proof of Theorem 2.5.7. We state the corresponding sequence of lemmas without proof. The central polygon is discussed in Proposition 2.5.24.

Lemma 2.5.17. Suppose that $X=E\left(x_{a}^{p}, x_{b}^{p+1}\right)$ and $Y=E\left(x_{i}^{p-1}, x_{j}^{p+1}\right)$ with $i>j$. Then the following are equivalent.

(1) $\mathcal{C}_{\Phi}(Y, X) \neq 0$

(2) $a<j \leq b$

(3) $\lambda_{1} X<\lambda_{1} Y-1 \leq \lambda_{2} X-1$

Lemma 2.5.18. Suppose that $X=E\left(x_{a}^{p-2}, x_{b}^{p-1}\right)$ and $Y=E\left(x_{i}^{p-1}, x_{j}^{p+1}\right)$ with $i>j$. Then the following are equivalent.

(1) $\mathcal{C}_{\Phi}(Y, X) \neq 0$

(2) $a<i \leq b$

(3) $\lambda_{1} X+1<\lambda_{2} Y \leq \lambda_{2} X$

In both lemmas, the three integers in (3) are congruent to each other modulo $m$. The condition $i>j$ is equivalent to $Y$ being $m+1$-rigid when $m \geq 5$.

Lemma 2.5.19. Let $Y=E\left(x_{i}^{p-1}, x_{j}^{p+1}\right)$ with $i>j$ and let $X$ be a standard indecomposable object.

(1) There exists $1 \leq k \leq m$ so that $X[k] \in C_{p, p+1}$ and $\operatorname{Ext}^{k}(Y, X) \neq 0$ if and only if

$$
\lambda_{1} X<\lambda_{1} Y<\lambda_{2} X
$$

(2) There exists $1 \leq k \leq m$ so that $X[k] \notin C_{p, p+1}$ and $\operatorname{Ext}^{k}(Y, X) \neq 0$ if and only if

$$
\lambda_{1} X<\lambda_{2} Y<\lambda_{2} X \text {. }
$$


Proposition 2.5.20. Suppose that $X, Y$ are $m+1$-rigid indecomposable objects where $X$ is standard and $Y$ is nonstandard. Then $X, Y$ are compatible if and only if neither $\lambda_{1} Y$ not $\lambda_{2} Y$ lies between $\lambda_{1} X$ and $\lambda_{2} X$. Equivalently, $X, Y$ are compatible if and only if $\Psi X, \Psi Y$ do not cross.

Lemma 2.5.21. Suppose that $Y=E\left(x_{i}^{p-1}, x_{j}^{p+1}\right), Z=E\left(x_{a}^{q-1}, x_{b}^{q+1}\right)$ where $i>j$ and $a>b$.

(1) Suppose $q=p$. Then $\mathcal{C}_{\Phi}(Y, Z) \neq 0$ iff $\lambda_{1} Y \leq \lambda_{1} Z$ and $\lambda_{2} Y \leq \lambda_{2} Z$.

(2) Suppose $q=p+1$. Then $\mathcal{C}_{\Phi}(Y, Z) \neq 0$ iff $\lambda_{2} Z<\lambda_{1} Y-1$.

Lemma 2.5.22. Suppose $m \geq 5$ and $Y, Z$ are nonstandard $m+1$-rigid indecomposable objects with $Y \in C_{p-1, p+1}$. Then there exists $1 \leq k \leq m$ so that $\mathcal{C}_{\Phi}(Y, Z[k]) \neq 0$ and $Z[k] \in C_{p-1, p+1} \cup C_{p, p+2}$ if and only if either $\lambda_{2} Y<\lambda_{2} Z$ and $\lambda_{1} Y<\lambda_{1} Z$ or $\lambda_{2} Z<\lambda_{1} Y$.

The same statement holds for $m=4$ if $C_{p-1, p+1}$ is construed to mean the set of all $E\left(x_{i}^{p-1}, x_{j}^{p+1}\right)$ with $i>j$ and similarly for $C_{p, p+2}$.

Using Serre duality to get the other two cases (when $Z[k] \in C_{p-2, p} \cup C_{p-3, p-1}$ ), we get that nonstandard $Y, Z$ are compatible if and only if all of the following hold:

(1) $\lambda_{2} Y \geq \lambda_{2} Z$ or $\lambda_{1} Y \geq \lambda_{1} Z$

(2) $\lambda_{2} Z \geq \lambda_{1} Y$

(3) $\lambda_{2} Z \geq \lambda_{2} Y$ or $\lambda_{1} Z \geq \lambda_{1} Y$

(4) $\lambda_{2} Y \geq \lambda_{1} Z$

By manipulating these inequalities we get the following.

Proposition 2.5.23. Suppose $Y, Z$ are nonstandard $m+1$-rigid indecomposable objects. Then $Y, Z$ are compatible if and only if one of the intervals $\left[\lambda_{1} Y, \lambda_{2} Y\right],\left[\lambda_{1} Z, \lambda_{2} Z\right]$ contains the other. Equivalently, $Y, Z$ are compatible if and only if $\Psi Y, \Psi Z$ do not cross.

Theorem 2.5.16 follows from Propositions 2.5.20 and 2.5.23.

Proposition 2.5.24. The central polygon in nonstandard $m$-cluster has $2 m-2$ sides. Given any nonstandard $m$-cluster $\mathcal{T}$ and object $T \in \mathcal{T}$, if $T$ is not a side of the central $2 m-2$-gon, then there are, up to isomorphism, exactly $m$ objects $T^{*}$ so that $\mathcal{T} \backslash T \cup T^{*}$ forms a nonstandard $m$-cluster. When $T$ is a side of the central $2 m-2$-gon then there are, up to isomorphism, exactly $3 m-6$ objects $T^{*}$ so that $\mathcal{T} \backslash T \cup T^{*}$ forms a nonstandard $m$-cluster. Furthermore, in both cases the objects $T^{*}$ are obtained as a sequence $T_{i}^{*}$ given by left $\mathcal{T} \backslash T$-approximation triangles $T_{i}^{*} \rightarrow B_{i} \rightarrow T_{i+1}^{*}$.

Proof. As in the proof of Theorem 2.5.7, we can contract all of the standard objects without changing the number of sides in the polygons having nonstandard sides. Then the central polygon has only two nonzero sides which are given by a single doubled nonstandard object $Y=E\left(x_{i}^{p}, x_{j}^{p+2}\right)$. We must have $i=j+1$ (otherwise, $E\left(x_{i}^{p}, x_{i-1}^{p+2}\right)$ would be compatible with all objects in $\mathcal{T})$. Then $\lambda_{2} Y-\lambda_{1} Y=m-2$ which means that the chord connecting the endpoints in $P_{\infty}$ has $m-1$ sides which means that the doubled object in $P_{\infty}^{(2)}$ has $2(m-1)$ sides.

When a side of this central $2 m-2$-gon is removed then two sides are removed by symmetry. So, we get a $6 m-10$-gon. which has $3 m-5$ trisections. So, there are $3 m-6$ mutations of any side. Figure 3 applies to this case and shows that the objects $T_{i}^{*}$ can be obtained by iterated approximation triangles. 
2.5.5. Example. For $m=5$, here is an example of a nonstandard $m$-cluster. Stardard objects are pairs of horizontal arc connecting $x_{i}^{p}$ to $x_{j}^{p+1}$ (given by consecutive letters in the notation of the figure). Nonstandard objects are pairs of vertical arcs connecting connecting $x_{i}^{p+2}$ on one side to $x_{j}^{p}$ on the other with $i<j$.

There is $2 m-2=8$-gon in center. Other regions have $m+2=7$ sides.

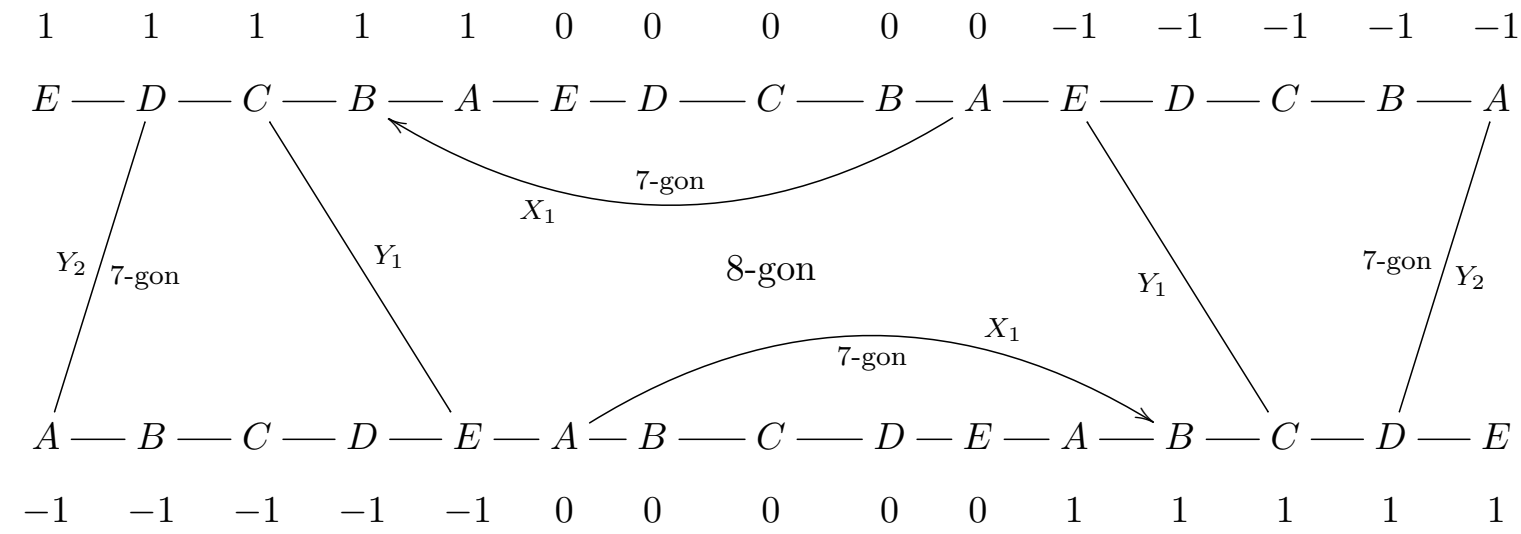

Standard: $X_{1}=E\left(A_{0}, B_{1}\right)=E\left(x_{0}^{1}, x_{1}^{2}\right)$ (horizontal).

$Y_{1}=E\left(C_{1}, E_{-1}\right)=E\left(x_{1}^{3}, x_{-1}^{5}\right), Y_{2}=E\left(D_{1}, A_{-1}\right)=E\left(x_{1}^{4}, x_{-1}^{1}\right)$ are nonstandard but $(m+1)$-rigid (vertical).

$x_{j}^{p}$ is the $p$ th letter of the alphabet with subscript $j$. For example, $x_{j}^{1}=A_{j}, x_{j}^{2}=B_{j}$.

We want to emphasize that the thick subcategory of standard objects in $\mathcal{C}_{\Phi}\left(Z_{\infty}^{m}\right)$ forms an $m$-cluster category in the usual sense. This example is illustrating the properties of the nonstandard objects in our category.

\section{REFERENCES}

[1] K. Baur, R. Marsh, A geometric description of m-cluster categories, Trans. Amer. Math. Soc. 360 (2008), no. 11, 5789-5803.

[2] A. Besser, A simple approach to geometric realization of simplicial and cyclic sets, E-print available at http://www.math.bgu.ac.il/bessera

[3] A. B. Buan, O. Iyama, I. Reiten, and J. Scott, Cluster structures for 2-Calabi-Yau categories and unipotent groups, Compos. Math. 145 (2009), no. 4, 1035-1079.

[4] Ragnar-Olaf Buchweitz, Maximal Cohen-Macaulay modules and Tate-cohomology over Gorenstein rings, preprint, 1986.

[5] Ragnar-Olaf Buchweitz, David Eisenbud, and Jürgen Herzog, Cohen-Macaulay modules on quadrics, Singularities, representation of algebras, and vector bundles (Lambrecht, 1985), Lecture Notes in Math., vol. 1273, Springer, Berlin, 1987, pp. 58-116.

[6] V. Drinfeld, On the notion of geometric realization, arXiv:math/0304064v3.

[7] Tobias Dyckerhoff, Compact generators in categories of matrix factorizations, Duke Math. J. 159 (2011), no. $2,223-274$.

[8] D. Eisenbud, Homological algebra on a complete intersection, with application to group representations, Trans. Amer. Math. Soc. 260 (1980), 35-64.

[9] D. Grayson, Algebraic K-theory, Lecture notes available at http://www.math.uiuc.edu/dan/Courses/2003/Spring/416/GraysonKtheory.ps

[10] Dieter Happel, Triangulated categories in the representation theory of finite dimensional algebras, London Math. Soc. Lecture Note Ser., vol. 119, Cambridge Univ. Press, Cambridge, 1988.

[11] T. Holm and P. Jørgensen, On a cluster category of infinite Dynkin type, and the relation to triangulations of the infinity-gon, Math. Z. 270 (2012), 277-295 
[12] _ Cluster tilting vs. weak cluster tilting in Dynkin type A infinity, Forum Math. DOI 10.1515/forum-2012-0093 (see http://www.degruyter.com/view/j/forum.ahead-of-print/forum-20120093/forum-2012-0093.xml).

[13] K. Igusa and G. Todorov, Continuous Frobenius categories, arXiv:1209.0038, to appear in Proceedings of the Abel Symposium 2011.

[14]_Continuous cluster categories I, arXiv:1209.1879.

[15] _ Continuous cluster categories of type D, arXiv:1309.7409.

[16] Group actions on Frobenius cyclic posets, in preparation.

[17] Peter Jørgensen, Auslander-Reiten theory over topological spaces, Comment. Math. Helv. 79 (2004), no. $1,160-182$.

[18] Mikhail Khovanov and Lev Rozansky, Matrix factorizations and link homology, Fund. Math. 199 (2008), no. 1, 1-91.

[19] Dmitri Orlov, Triangulated categories of singularities and D-branes in Landau-Ginzburg models, Tr. Mat. Inst. Steklova 246 (2004), no. Algebr. Geom. Metody, Svyazi i Prilozh., 240-262.

[20] _ Matrix factorizations for nonaffine LG-models, Math. Ann. 353 (2012), no. 1, 95-108.

[21] Adam-Christiaan van Roosmalen, Hereditary uniserial categories with Serre duality, Algebr. Represent. Theory 15 (2012), no. 6, 1291-1322.

Department of Mathematics, Brandeis University, Waltham, MA 02454

E-mail address: igusa@brandeis.edu

Department of Mathematics, Northeastern University, Boston, MA 02115

E-mail address: g.todorov@neu.edu 